\title{
Detached eddy simulation of turbulent flow around square and circular cylinders on Cartesian cut cells
}

\author{
W. Bai ${ }^{\text {a, }}$, C.G. Mingham ${ }^{\text {b }}$ D.M. Causon ${ }^{\text {b }}$ L. Qian ${ }^{\mathrm{b}}$ \\ a Department of Civil and Environmental Engineering, National University of Singapore, Kent Ridge, Singapore 117576, \\ Singapore \\ b School of Computing, Mathematics and Digital Technology, Manchester Metropolitan University, Chester Street,
} Manchester M1 5GD, UK

\begin{abstract}
Square and circular cylinders in three-dimensional turbulent flows are studied numerically using the LES and DES turbulence models. One aim of the present study is to implement the LES and DES turbulence models in a cell-centered finite volume method (FVM) developed for solving the Navier-Stokes equations on Cartesian cut cells. The Cartesian cut cell approach is known to be robust for problems in geometrically complex domains with fixed or moving boundaries. For the purpose of validating the present numerical model, the current flow past fixed square and circular cylinders at moderate Reynolds numbers is tested first. Comparison of the computed results with experimental data reveals that the DES models are superior to the conventional LES and RANS models. The second aim of the present study is to assess the performance of different RANS based DES turbulence models. By means of the comparison of results obtained with the 0-equation mixing-length, 1-equation S-A and 2-equation $k$ - $\omega$ based DES models for the flow over the same circular cylinder, some recommendations are proposed. According to the present study, in terms of accuracy the 1-equation S-A based DES model is very promising. Beside this, if the computational cost is the main concern, the 0-equation mixing-length based DES model might be an ideal option, achieving a good balance between accuracy and efficiency.
\end{abstract}

Key words: LES model; DES model; Turbulent flow; Circular cylinder; Square cylinder; Cartesian cut cell

\section{Introduction}

Most flows of significant engineering relevance are turbulent flows dominated by large-scale unsteadiness and coherent vortex shedding. To successfully simulate such kinds of flows is a big challenge, especially when the computational domain is complex or a moving boundary is considered. Due to the dramatic progress in computer technology, computational fluid dynamics (CFD) is now able to handle such industrially relevant flows at moderate costs. At the present time, there have been several different computational approaches for simulating turbulent flows. The most accurate approach to turbulence

\footnotetext{
* Corresponding author.

E-mail address: w.bai@nus.edu.sg (W. Bai).
} 
simulation is to solve the Navier-Stokes equations directly, without averaging or approximation [1-4]. This direct numerical simulation (DNS), in principle, is the only method capable of capturing all aspects of turbulence. However, the direct resolution of the turbulent motion is infeasible, as the associated grid resolution would cause prohibitive computational expenses. Therefore, most of the current DNS applications are aimed at the simulation of flows at low Reynolds numbers. In the foreseeable future, it is likely that computers will not be able to meet the challenge of a high Reynolds number calculation using the method of DNS.

In the past, the Reynolds-averaged Navier-Stokes equations (RANS) seemed to be the only option to calculate turbulent flows of industrial interest $[5,6]$. In the framework of RANS, all aspects of turbulence are modelled, which enhances the numerical efficiency at the expense of a strong model dependency. Generally speaking, RANS models work well in flows in which slow varying coherent structures contribute a considerable portion of the total turbulence kinetic energy [7]. Due to the fact that a statistical or temporal average is being adopted, RANS calculations often fail to capture unsteady flow phenomena in the wake behind bluff bodies or airfoils at high angles of attack. On the other hand, large eddy simulation (LES) of turbulent flows is thought to be the most accurate method for high Reynolds numbers which exceed the present DNS capability. LES is much less sensitive to modelling errors since only the small subgrid scales of motion are modelled. However, LES demands very fine near-wall resolution to directly resolve the turbulent structures. This is basically due to the absence of universal wall functions, which would allow for a reduced number of grid points in the near-wall region [8]. For this reason, wall-resolving LES remains fairly time consuming, disqualifying this method for industrial applications especially at higher Reynolds numbers. Some successful results with LES have been reported by Kitagawa and Ohta [9] and Liang and Papadakis [10] on one or two stationary circular cylinders in a cross-flow.

Recently, the detached eddy simulation (DES) has become a promising tool for the prediction of turbulence as it offers a reduced computational effort in comparison to LES while retaining much of the physical accuracy of the method. The DES was first proposed by Spalart et al. [11] in 1997 based on the Spalart-Allmaras (S-A) eddy-viscosity RANS model [12]. This was followed by a more general discussion in Travin et al. [13]. The DES method aims to combine the fine-tuned RANS methodology in the attached boundary layers with the power of LES in the shear layers and separated flow regions, thereby considerably reducing the cost of the computations. Unlike other hybrid models which explicitly divide the solution domain into distinct RANS and LES regions with matching of the two different models being an issue, the DES method provides a seamless transition between the two regions by adopting a single turbulence model. To date, there have been a number of successful DES applications ranging from classical configurations such as the flow over a cylinder and sphere to complex geometries including fighter aircraft [14-17].

From the above discussion, it may be observed that the DES is constructed on the basis of a certain RANS turbulence model. However, different types of RANS models are available currently. In short, we can classify them into 0 -equation model, 1-equation model and 2-equation model, according to the number of additional transport equations to be solved in the RANS models. In this paper, the different RANS based DES turbulence models will be evaluated, using the example of flow over a circular cylinder. Although 
detailed comparisons of the different turbulence models have been reported in the past, such as the comparison of LES, RANS and DES models for flows past cubes and spheres [18] or a flat plate [19], similar comparisons between different RANS based DES models have, to the best of the authors' knowledge, yet appeared in the literature for any flow problem. In the following, the 0-equation RANS model refers to the mixing-length turbulence model, and the 1-equation and 2-equation models are chosen as the widely used S-A and $k$ - $\omega$ turbulence models respectively.

The aims of the present paper are twofold. The first is to implement the different turbulence models in a numerical Navier-Stokes solver based on the Cartesian cut cell approach. This approach is an effective alternative to traditional structured and unstructured grids. Solid regions are simply cut out of a stationary background Cartesian mesh, and their boundaries are represented by different types of cut cells. Therefore, a single Cartesian mesh is composed of solid cells, fluid cells and partially cut cells. The cut cell mesh generation is relatively straightforward through calculations for the boundary segment intersections with the background Cartesian mesh. Furthermore, moving boundaries can be easily accommodated by re-computing cell-boundary intersections, rather than re-meshing the whole flow domain or large portions of it. For this reason, the Cartesian cut cell approach is very suitable for complex computational domains or moving boundaries that exist in a broad class of engineering problems. This method has recently been applied successfully to the shallow water equations [20, 21], and extended to deal with incompressible viscous flows [22, 23]. In the present paper, the different turbulence models, including the Smagorinsky-type LES model, the 0-equation, 1-equation and 2-equation RANS models and the corresponding DES models are incorporated into this robust Navier-Stokes solver. The developed numerical model is verified by comparisons with experimental data for flow over square and circular cylinders. The validation indicates the effectiveness of the DES turbulence model, when it is even constructed on the basis of the simplest 0-equation RANS model.

The second aim is to carry out the aforementioned comparison between the different RANS based DES models. Through the comprehensive comparison of the mean velocity and Reynolds stress components in the wake behind the circular cylinder, particular DES turbulence models are identified as the better options for this case, in terms of accuracy and efficiency.

\section{Mathematical formulation}

The conventional numerical approaches to turbulent flows are the RANS and the LES methods, both of which need to solve the averaged Navier-Stokes equations. However, the average is taken over a certain time period in RANS, while it is a spatial average over a small volume in LES. Due to the nonlinearity of the Navier-Stokes equations, models are needed in order to close the averaged equations, which are termed turbulence models in RANS and subgrid scale models in LES.

\subsection{Smagorinsky LES turbulence model}


In the LES method, a space filter function is used to filter the continuity and Navier-Stokes equations. The filtered governing equations then read

$$
\begin{aligned}
& \frac{\partial \bar{u}_{j}}{\partial x_{j}}=0 \\
& \frac{\partial \bar{u}_{i}}{\partial t}+\bar{u}_{j} \frac{\partial \bar{u}_{i}}{\partial x_{j}}=-\frac{1}{\rho} \frac{\partial \bar{p}}{\partial x_{i}}+v \frac{\partial^{2} \bar{u}_{i}}{\partial x_{j} \partial x_{j}}-\frac{\partial}{\partial x_{j}}\left(\overline{u_{i} u_{j}}-\bar{u}_{i} \bar{u}_{j}\right)+g_{i}
\end{aligned}
$$

where the overbar denotes the filtered (resolvable) quantities, $u_{i}$ is the filtered velocity, $\rho$ and $v$ are the density and the kinematic viscosity of the fluid, $g$ is the acceleration due to gravity and $p$ denotes the pressure. The new term appearing in the filtered equations is:

$\tau_{i j}=-\left(\overline{u_{i} u_{j}}-\bar{u}_{i} \bar{u}_{j}\right)$

where $\tau_{i j}$ is called the subgrid scale (SGS) Reynolds stress. It is noticed that $\tau_{i j}$ is similar to the RANS Reynolds stress, but the physics it represents is different. In RANS models, all the turbulent motions are modeled, while in LES models, only the turbulent motions smaller than the filter size (subgrid scale) are modeled and the motions larger than the filter size (large eddies) are explicitly computed. The large scale motions are affected by the flow geometry and are different from flow to flow, but the small scale motions are more universal. Therefore, it is reasonable to expect that the model closure for LES models is less demanding than that for RANS models, and LES models are more accurate and reliable than RANS models especially for flows in which large-scale unsteadiness is significant.

Using the Boussinesq assumption, we obtain the final form of the space filtered Navier-Stokes equations,

$$
\frac{\partial \bar{u}_{i}}{\partial t}+\bar{u}_{j} \frac{\partial \bar{u}_{i}}{\partial x_{j}}=-\frac{1}{\rho} \frac{\partial \bar{p}}{\partial x_{i}}+\frac{\partial}{\partial x_{j}}\left[\left(v+v_{T}\right) \frac{\partial \bar{u}_{i}}{\partial x_{j}}\right]+g_{i}
$$

where $v_{T}$ is the SGS eddy viscosity. Now, we need a turbulence closure to model the unresolved scale (SGS) motions. The most basic subgrid scale model is the one proposed by Smagorinsky in 1963, which is called the standard Smagorinsky model. In this turbulence model, the form of the subgrid scale eddy viscosity can be derived by dimensional arguments and is

$v_{T}=\left(C_{S} \Delta\right)^{2}\left(2 S_{i j} S_{i j}\right)^{1 / 2}$

where $C_{S}$ is the Smagorinsky constant, $\Delta$ is the characteristic subgrid length scale given by

$\Delta=\left(\Delta_{x} \Delta_{y} \Delta_{z}\right)^{1 / 3}$

in which $\Delta x, \Delta y$ and $\Delta z$ are the grid sizes in the $x, y$ and $z$ directions respectively, and $S_{i j}$ is the shear strain rate tensor for the resolved scales,

$S_{i j}=\frac{1}{2}\left(\frac{\partial \bar{u}_{i}}{\partial x_{j}}+\frac{\partial \bar{u}_{j}}{\partial x_{i}}\right)$

One of the disadvantages of this model is that the value of $C_{S}$ is dependent on the flow type and it may be necessary to choose different values in different flow zones. Commonly, the widely used value of $C_{S}$ varies from 0.1 to 0.3 , and is chosen here to be 0.1 , as in [ 8 and 9 ]. 


\subsection{Concept of DES model}

In order to obtain the model used in the DES formulation, the corresponding turbulence length scale $l_{R A N S}$ in the individual RANS turbulence model has to be specified first. This length scale is then modified to ensure it is no greater than the local grid spacing, i.e.

$d_{D E S}=\min \left(l_{R A N S}, C_{D E S} \Delta\right)$

where $d_{D E S}$ is the DES length scale which is adopted in solving the RANS type of turbulence equations in the DES model. Here $C_{D E S} \Delta$ is the local grid length scale, and $C_{D E S}=0.65$, as recommended in $[8,17$ and 24]. $C_{D E S} \Delta$ takes the role of the filter width in the LES mode, as it is directly based on the local grid dimension. The aim of the DES model is to use a RANS simulation in the vicinity of walls, and LES in areas of massive flow separation outside of the boundary layer. The RANS models are usually calibrated and well understood for wall-bounded flows, which underscores the basic motivation for the DES approach.

By defining the modified turbulence length scale in Eq. (8), only the RANS equations need to be solved in the entire computational domain, without any explicit distinction between RANS and LES zones. The change in the length scale leads to a model that becomes region-dependent in nature: in most cases a RANS model in the boundary layers and a LES model in separated regions. These advantages of the DES model can help to increase the efficiency of the numerical method significantly, because normally only a relatively coarse mesh is required in the boundary layer around the walls, instead of the very fine mesh used in the LES model.

\subsection{Different RANS based DES models}

In RANS models, due to the ensemble averaging process, further unknowns are introduced in the momentum equations by means of the Reynolds stresses, which are typically modelled by employing the Boussinesq-viscosity hypothesis for linear eddy viscosity models. Consequently, the governing equations can be reduced to the same form as in the LES model (Eq. 4), as discussed before. There are several turbulence models available to predict the eddy viscosity in Eq. (4), ranging from the simplest 0-equation mixing-length model to the 1-equation S-A model and the 2-equation $k-\omega$ model. The implementation of these RANS models in the detached eddy simulation is discussed next.

In the 0-equation mixing-length turbulence model, one can express the eddy viscosity as the product of a velocity scale and a length scale, in which the appropriate specification of the mixing length $l_{m-l}$ is

$l_{m-l}=\kappa d$

where $\kappa=0.41$ is the Von Karman constant, and $d$ is the distance to the nearest wall. It should be noted that to apply the mixing-length turbulence model in the DES, $l_{m-l}$ should be substituted into Eq. (8) representing $l_{R A N S}$ to determine the DES length scale $d_{D E S}$. As a result, $d_{D E S}$, instead of $l_{m-l}$, will be used to calculate the 
eddy viscosity $v_{T}$ following the same equation in the conventional mixing-length RANS model,

$v_{T}=\left(d_{D E S}\right)^{2}\left(2 S_{i j} S_{i j}\right)^{1 / 2}$

The DES length scale $d_{D E S}$ predicted from Eq. (8) can switch automatically between the RANS length scale and the local grid length scale according to the position in the flow field. Although it is very easy to implement and fast to calculate, the conventional mixing-length turbulence model is known to be completely incapable of describing flows where the turbulence length scale varies and separation or recirculation exist. By using the DES concept, the mixing-length turbulence model can be transformed to the subgrid scale model where necessary, in order to overcome its original disadvantage.

The S-A model proposed in [12] is another turbulence model, which requires solving one additional transport equation. This transport equation contains convective and diffusive transport terms, as well as expressions for the production and dissipation of the eddy viscosity. In this model, the eddy viscosity $v_{T}$ is obtained from a viscosity-like variable $\tilde{r}$,

$v_{T}=\tilde{1} \ldots \ldots=\frac{\chi^{3}}{\chi^{3}+C_{v 1}^{3}}, \quad \chi=\frac{\tilde{r}}{v}$

and the turbulence length scale $l_{S-A}$ for this case is the distance $d$ to the nearest wall. The transport equation for this viscosity-like variable $\tilde{r}$ has the following form, making use of the concept of the DES model,

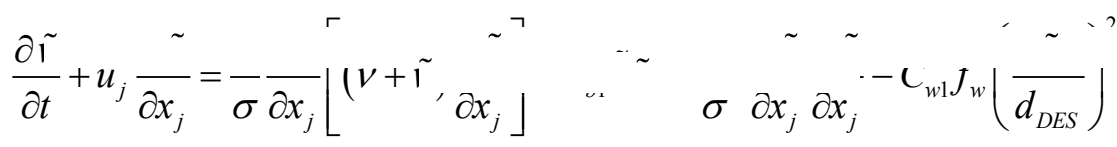

The modified magnitude of the strain rate $\tilde{i}$ is expressed as

$$
\tilde{i} \quad\left({ }^{---_{l}-L_{i j}}\right)^{1 / 2}+\frac{\tilde{r}}{\kappa^{2} d_{D E S}^{2}} J_{v 2}, f_{v 2}=1-\frac{\chi}{1+\chi f_{v 1}}
$$

where $\Omega_{i j}$ is the magnitude of the vorticity. The function $f_{w}$ in Eq. (12) is given by

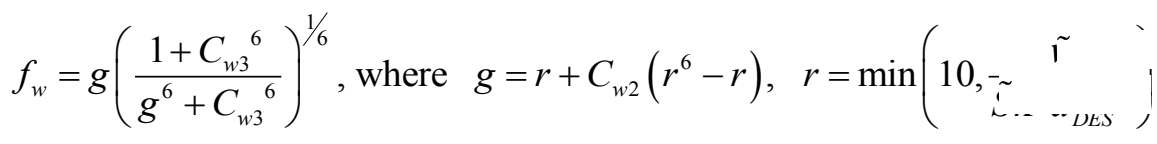

The constants in the above equations are $C_{v 1}=7.1, \sigma=2 / 3, C_{b 1}=0.1355, C_{b 2}=0.622, C_{w 1}=3.239, C_{w 2}=$ 0.3 and $C_{w 3}=2$. The S-A model is economical and accurate for attached wall-bounded flows, but weak for massively separated flows. However, the use of the DES concept is expected to avoid the abovementioned weakness.

The two-equation turbulence models have gained the most popularity in industrial applications. The present study is confined to the standard $k-\omega$ model of Wilcox [25]. It was claimed there that the model can be extended to the near-wall region without any kind of damping functions. The model was reported to perform notably better in transitional flows and in adverse pressure gradient boundary layer flows. In this model, a turbulence specific dissipation rate $\omega$ is defined as $\omega=\varepsilon /\left(C_{\mu} k\right)$, replacing the dissipation rate $\varepsilon$ in the $k-\varepsilon$ model. Here, $k$ is the turbulent kinetic energy and $C_{\mu}$ is a constant and equal to 0.09 [5]. Based on the definition of $\omega$, the determination of eddy viscosity needs to be changed accordingly to $v_{T}=k / \omega$. 
Based on the strategy of the DES model, the turbulence length scale of the standard $k$ - $\omega$ model should be determined first, which has the form,

$l_{k-\omega}=\frac{k^{0.5}}{C_{\mu} \omega}$

After comparing with the local grid size, the length scale of the DES model $d_{D E S}$ can be predicted by Eq. (8). Consequently, the transport equation in Wilcox [25] for the turbulent kinetic energy $k$ can be rearranged, in conjunction with the length scale of the DES model obtained before, leading to

$\frac{\partial k}{\partial t}+u_{j} \frac{\partial k}{\partial x_{j}}=\frac{\partial}{\partial x_{j}}\left[\left(v+\frac{v_{T}}{\sigma_{k 1}}\right) \frac{\partial k}{\partial x_{j}}\right]+P_{k}-\frac{k^{1.5}}{d_{D E S}}$

The transport equation for the specific dissipation rate $\omega$ remains unchanged in the $k$ - $\omega$ based DES turbulence model, and its standard expression is

$\frac{\partial \omega}{\partial t}+u_{j} \frac{\partial \omega}{\partial x_{j}}=\frac{\partial}{\partial x_{j}}\left[\left(v+\frac{v_{T}}{\sigma_{w 1}}\right) \frac{\partial \omega}{\partial x_{j}}\right]+\gamma_{1} \frac{\omega}{k} P_{k}-\beta_{1} \omega^{2}$

The model closure coefficients used for the $k$ - $\omega$ turbulence model [25] are $\sigma_{k 1}=2.0, \sigma_{\omega 1}=2.0, \gamma_{1}=0.553$ and $\beta_{1}=0.075 . P_{k}$ denotes the production of turbulent energy, which can be taken the form as $P_{k}=\tau_{i j} \partial u_{i} / \partial x_{j}$ $=v_{T}\left(2 S_{i j} S_{i j}\right)$.

\subsection{Boundary conditions}

Besides satisfying the governing momentum and continuity equations, the flow is also subject to various boundary conditions on all surfaces of the fluid domain, as shown in Fig. 1. On the solid body surfaces non-slip boundary conditions are applied, and the fluid velocity is specified at the inlet boundary. At the outlet boundary, the set condition should make the boundary "transparent", i.e. the numerical solutions in the inner computational region should not be affected by the outlet boundary. At the far field boundary, a zero normal velocity gradient is implemented [5, 24], which is found to be effective as no obvious reflection has been detected in the numerical simulations. The distance from the cylinder to the outlet boundary is sufficiently large, which further ensures that the outlet boundary has a negligible influence on the near-wake region of the flow. A Neumann-type boundary condition needs to be introduced for the pressure on all the boundary surfaces. Furthermore, the boundary conditions also need to be set for various unknown turbulence variables. At all non-slip boundaries, the turbulent kinetic energy and the specific dissipation rate are all taken to be zero, i.e. $k=\omega=0$.

Since the problem is solved in the time domain, an initial condition must be imposed. In order to accelerate the speed of convergence, the free stream velocity $u_{0}$ at the inlet boundary is used to prescribe the fluid velocity within the whole domain. The turbulence variables are given a uniform value all over the domain as $k=1 \times 10^{-6} u_{0}^{2}$ and $\omega=10 \mathrm{k} / \mathrm{v}$. At the same time, it is often assumed that the flow in local equilibrium, meaning the production and dissipation of $k$ and $\omega$ are nearly equal. Based on this approximation, the equation for $\omega$ is not applied in the elements next to the wall [26]; instead, $\omega$ at these 
elements is set equal to:

$$
\omega=\frac{k^{0.5}}{C_{\mu}{ }^{0.25} \kappa d}
$$

Fig. 1. Definition sketch of the flow domain

\section{Numerical method}

A numerical solution to the Navier-Stokes questions has been developed by using a cell-centered Finite Volume Method (FVM) based on a Cartesian cut cell approach in Bai et al. [23], where applications have been carried out for 2D laminar flows with and without free water surfaces. This well-established algorithm is of second-order accuracy both in space and time, and it is extended to deal with the turbulent flows in this paper. The cut cell method produces a boundary conforming mesh without the necessity to make the boundary a coordinate surface. In fact, there is no mesh generation in the conventional sense; all that is necessary is to calculate the intersections of a series of line segments with a background Cartesian grid. Since, in practice, a cut cell can be arbitrarily small, numerical stability may be compromised at these small cut cells. To overcome this problem cell merging is implemented. Therefore, the total computational domain is composed of fluid cells, cut cells, solid cells, merged cells and small merging cells, as shown in Fig. 2(a). It should be noted that Fig. 2(a) is just a sketch to show part of a body surface. The arrow in the figure defines a direction, by which the solid phase is always located on the left hand side of the line segment. Fig. 2(b) shows the example cut cell mesh around a circular cylinder, from which we can see finer grids are adopted in the region close to the body. The intersection points between the body boundary and the background grid are clearly shown, and different cut cells and merged cells can be identified. Only fluid cells, cut cells and merged cells need to be treated separately; these will be discussed next.

Fig. 2. (a) Sketch of definition for the cut cell mesh; (b) example cut cell mesh around a circular cylinder

One important advantage of the Cartesian cut cell approach is that it is convenient to apply on the present cut cells the well-tested flow solvers that were originally developed for other types of grids; only slight modifications for cut cells and merged cells are required, as indicated in Bai et al. [23]. In other words, except for the equations given in the last section, no additional mathematical equations for both the flow field and the turbulence model are introduced in the Cartesian cut cell approach. In this paper, for fluid cells the momentum equation is discretized by second-order accurate schemes, and the SIMPLE algorithm with a collocated variable arrangement is adopted to solve the pressure-velocity coupling; more details can be found in many textbooks, such as Ferziger and Perić [26].

For cut cells, the discretized equation is based on the same algorithm developed for fluid cells, with the 
non-slip boundary condition at the cut surface corresponding to the solid wall. However, when the neighboring cell of a cut cell is a small merging cell (such as the triangular cell above the cut cell shown in Fig. 2a), a specific explicit treatment is used to avoid arranging unknowns on the small merging cell, because in the small merging cell a much smaller time step is required to ensure the numerical stability. In this case, the values of the properties in the small merging cell are known from the previous iteration. For merged cells, the situation is more complex, because two neighboring cells may exist at one cell face of a merging cell (such as the bottom faces of the merged cells shown in Fig. 2a). When this situation happens, one neighboring cell is a fluid cell and the other one is a cut cell or a small merging cell, see Bai et al. [23] for more details.

By means of the SIMPLE algorithm, an iteration procedure can finally provide the velocity and the pressure of the fluid at a certain time instant once convergence has been achieved. When the LES turbulence model is adopted, at the end of each time instant, the eddy viscosity needs to be updated by using Eq. (5). The 0-equation based DES model is similar to the LES model, because in neither of them it is necessary to solve additional transport equations: the eddy viscosity is calculated using Eq. (10). If the 1-equation or 2-equation RANS based DES turbulence model is involved in the simulation, we have to solve one or two additional transport equations, Eq. (12) or Eqs. (16) and (17), which have a very similar formulation to the momentum equation in Eq. (4). Therefore, similar numerical schemes in the momentum equation discussed above can be used to discretize the transport equations in the RANS based DES models. In addition, in order to ensure the stability of the simulation, this solving process is embedded into the SIMPLE iteration procedure, which means the turbulence transport equations need to be solved and the eddy viscosity updated immediately after the calculation of the flow field in each iteration within one time step. In sum, our algorism at one particular time step proceeds as follows: 1. Solve the moment equations explicitly to obtain the velocity; 2. Calculate the pressure correction by solving the pressure correct equation, by which the pressure and the obtained velocity are corrected; 3 . Check the convergence of the calculation, and go to Step 6 if the convergence is achieved; 4. Solve the addition transport equations if the 1-equation or 2-equation RANS based DES model is adopted, and update the eddy viscosity; 5. Return to Step 1 for the next iteration; 6 . Update the eddy viscosity for all various turbulence models, and proceed to the next time step.

\section{Numerical results}

In this section, the turbulent flows past a square cylinder and a circular cylinder are investigated using the present numerical model. These two cases feature geometry-fixed and pressure-induced separation, impinging flow and streamline curvature. The numerical results obtained by using different turbulence models are compared with the experimental data for these two cases, which indicates the accuracy and effectiveness of the DES model performed on Cartesian cut cells, despite it being based on a very simple RANS turbulence model. In addition, an assessment of performance of the different RANS based DES models is made. 
It should be noted that all the examples included in this paper only consider turbulent flow at moderate Reynolds numbers, ranging from 3900 to 14000 . The focus of the present study is placed on the implementation and assessment of different turbulence models; therefore, validation of the developed numerical model is critical. This can be achieved by comparing with reliable experimental data at moderate Reynolds numbers. In principle, the current numerical model is able to handle flows at higher Reynolds numbers, as long as the mesh and time step are fine enough. The Reynolds number $(\mathrm{Re})$ is defined in terms of the typical body size $D$ that here is taken as the length of the edge for a square cylinder or the diameter for a circular cylinder, respectively. The fluid density $\rho$, free stream velocity $u_{0}$ and typical body size $D$ are all taken to be unity in all cases discussed below, helping to yield results in non-dimensional form. The simulations are performed in a rectangular domain $20 D$ long and $14 D$ wide, and the body centers are located $5 D$ to the left-hand inlet boundary and $7 D$ to the bottom boundary, as shown in Fig. 1.

The abovementioned rectangular domain refers to the two-dimensional plane (the $x-y$ plane) which is perpendicular to the axis of the cylinder. The Cartesian cut cell meshes are generated in this plane, and the three-dimensional grid is then created by simply extending the two-dimensional meshes in the direction along the axis of the cylinder (the $z$ direction). The length of the domain in the $z$ direction is set at $2 D$ with slip boundary conditions imposed on the ends. For most of the computations, 15 grid points are uniformly located in that direction. However, 30 grid points are also used in some cases in order to test the convergence of the computations. On the $x-y$ plane, a non-uniform grid is generated, in which a relatively coarser mesh is employed in the region far away from the body, while a finer mesh must be generated around the body surface. In addition, a fine mesh is also required in the wake behind the cylinder, and this zone is $4.5 D$ in length measured from the center of the cylinder. The present study focuses on the investigation of flow properties of engineering interest, such as the global hydrodynamic characteristics and turbulent flow structures in the near wake. Extensive comparison with the experimental data is carried out, by which the performance of the present numerical model can be evaluated.

\subsection{Flow around a square cylinder at $R e=14000$}

The turbulent flow over a square cylinder is one key flow configuration for exploring the validation of different turbulence models. When the oncoming laminar flow is impinging on the cylinder front, it is known that the flow separates at the leading edges, and undergoes transition to turbulence in the separated shear layers and forms a vortex street in the wake due to periodic vortex shedding at the lee side of the cylinder. The Reynolds number being considered here is 14000 , based on the availability of experimental data for comparison. For this case, the influence of mesh refinement is first investigated, mainly using three grids referred to as "Mesh0", "Mesh1" and "Mesh2" below, as shown in Table 1. Moreover, a grid with more points in the $z$ direction is also tested, which is defined as "Mesh1_NZ". Different turbulence models have been adopted on the above grids. To test the effectiveness of the DES concept, we begin with the LES and a DES model based on the very simple mixing-length model denoted as "LES" and "MLD" respectively. The results of the mixing-length RANS model are also included for the purpose of comparison, 
with the notation "MLM" in Table 1. It should be noted that the total number of cells may not change much for these three grids. However, the minimum grid size around the body surface is most important for the test of mesh convergence, which varies from 1.44 to 1.0. The numerical results suggest that this range of grid sizes illustrates the convergent trend of the calculations.

Table 1. Mean drag coefficient and Strouhal number of flow over the square cylinder

Table 1 shows the global flow characteristics obtained by the numerical simulations using different turbulence models on the various grids. In the table, the first column is the notation for different experimental and computational cases, and the following three columns represent the numbers of cells in the $x, y$ and $z$ directions, the total number of cells in the computational domain and the minimum grid size $\Delta_{\min }$ around the square cylinder. The drag coefficient $\left(C_{D}\right)$ and the Strouhal number (St) are shown in the last two columns, which are also compared to the data from the physical experiments conducted by Norberg [27] and Durao et al. [28] for the same Reynolds number.

From the table, it can be seen that the drag coefficient predicted by the LES converges fast with the grid. The coarse mesh "Mesh0" cannot provide the correct drag coefficient, whereas no much difference can be detected from the results using the other grids. In addition, more grid points in the $z$ direction seem to have little influence on the drag coefficient. On the other hand, the conventional mixing-length RANS turbulence model can predict the drag coefficient well, even with the coarse mesh. Unfortunately, this RANS model is not able to capture the unsteadiness of the flow field, as indicated in the column for the Strouhal number. However, the introduction of the DES concept can significantly improve the prediction of the Strouhal number, which indicates that switching to DES automatically leads to superior results of the flow field compared to the corresponding RANS model. In addition to this, the DES model can also provide better results compared to the LES model on the same coarse grid "Mesho", and this demonstrates the main advantage of the DES model. It is noticeable that the DES simulation is convergent on "Meshl", but it over-predicts the drag force, which reveals that the present DES model may not be very capable of fully resolving the boundary layer. However, in the present flow regime where the boundary layer remains fully laminar, the use of a non-refined near wall spatial resolution is acceptable by assuming that influence of the detailed structure of the boundary layer on the far wake is rather weak. The conjecture will be examined in the following by many comparisons between experimental and numerical results. Furthermore, in the calculations presented in this paper, the dimensionless time interval $\Delta t$ is set to 0.05 . In order to test the convergence of the calculation with the time interval, one additional case with a smaller dimensionless time interval of $\Delta t=0.02$ is conducted, which is denoted as "MLD_Mesho_ $\Delta t$ " in the table. The result indicates that the calculation is not very sensitive to the time interval, as long as the stable result is achieved.

Fig. 3 shows the time history of the lift and drag coefficients respectively, which are obtained using the simplest mixing-length based DES turbulence model on the coarse grid "Mesh0". It can be seen from the 
figure that the lift force is oscillating regularly at the natural vortex shedding frequency, whereas many irregular higher-frequency components can be found in the drag force, indicating the drag force is more sensitive to the turbulence effects.

Fig. 3. Time history of drag and lift coefficients on the square cylinder for Case MLD_Mesh0

The statistics of the flow variables from the current simulations are then compared to the results of the previous experimental study. The statistics are accumulated over approximately the last 20 vortex shedding periods, i.e. after $t=150$. The mean velocities and turbulent Reynolds stresses on the center line in the wake are shown in Fig 4 and Fig. 5. Obviously, the LES model on the coarse grid "Mesho" cannot provide accurate results because the grid resolution is not good enough to capture the flow phenomena. However, on the same grid, the performance of the mixing-length based DES model is much better than the LES model, which is very close to the LES model on the fine mesh "Meshl". A closer look at the centerline profiles of the mean streamwise velocity component reveals that both LES and DES models yield a smaller recirculation region behind the square cylinder at $x / H<2$, which may be due to the influence of the boundary layer. In addition, the mean streamwise and spanwise Reynolds stress components seem to have the same amplitude as the experimental data.

Fig. 4. Mean streamwise velocity component on the center line in the wake of the square cylinder

Fig. 5. Mean streamwise and spanwise Reynolds stress components on the center line in the wake of the square cylinder: (a) streamwise; (b) spanwise

Fig. 6 is the mean pressure coefficient around the square cylinder, and its comparison with the experimental data in Norberg [27], and Bearman and Obasaju [29] designated BO82 in the figure. Again, the numerical results from both the mixing-length based DES model on the coarse grid and the LES model on the fine grid agree fairly well with the experimental data, but the LES model on the coarse grid fails to do so. In addition, this figure clearly shows the pressure difference between the front (0-1) and back (2-3) edges of the square cylinder, which causes the drag force.

Fig. 6. Comparison of pressure coefficient around the square cylinder

\subsection{Flow around a circular cylinder at $R e=3900$}

The flow around a circular cylinder at a subcritical Reynolds number is another benchmark for turbulence models on the way towards their application for complex flows of engineering relevance. Due to the availability of measurements of the mean flow field in the very near wake by Lourenco and Shih [30], 
Beaudan P and Moin [31] and in the near wake by Ong and Wallace [32], the flow at $\mathrm{Re}=3900$ is studied in this section. Unlike the case of a square cylinder, impinging issues are less severe here, because of the streamlined shape of the obstacle. However, the separation of the pressure driven boundary layer from the continuous surface of a circular cylinder is a much more delicate task, and the location of the separation point depends on the details of the attached boundary layer.

The averaged drag coefficient and the Strouhal number for different grids are summarized in Table 2 . The comparison with the experimental results obtained by Norberg [33] leads to many conclusions similar to those we have drawn in Table 1 for the flow over a square cylinder. In short, this highlights again that the drag coefficient and the Strouhal number predicted by the mixing-length based DES model on the coarse grid agree better with the measurements in the experiments, but the drag coefficient when convergent is slightly larger than the experimental data. In addition, the similar convergence test with the time interval is carried out for the flow past the circular cylinder, and the numerical result indicates again that the time step adopted is small enough to ensure the solutions are time-step independent.

Table 2. Mean drag coefficient and Strouhal number of flow over the circular cylinder

The present mean velocities are compared with the results of the particle image velocimetry experiment of Lourenco and Shih [30] designated LS93 in the figure in the very near wake (up to $x / D=4$ ) and with the hot-wire measurements of Ong and Wallace [32] designated OW92 in the figure in the downstream region (from $x / D=3$ to $x / D=10$ ). Fig. 7 shows the mean streamwise velocity component on the center line in the wake. In the physical experiment, the recirculation region appears approximately up to $x / D=1.7$ behind the circular cylinder. However, we can notice that both the LES model and the mixing-length based DES model over-predict the recirculation region on the coarse grid, although the result from the DES model is closer to the experimental data than that from the LES model. In addition, good agreement between the simulation and the experiment can be achieved when the LES is implemented on a fine grid. The mean streamwise and spanwise velocity components at five different stations, $x=1.06, x=2.02, x=3.0, x=4.0$ and $x=5.0$, are plotted in Fig. 8 from the left to the right respectively. Generally speaking, the results of the LES on the fine grid and the DES on the coarse grid are very close to each other. At the same time, both of them also agree well with the experiment, except the spanwise velocity component in the very near wake at $x=1.06$. For the purpose of demonstrating this discrepancy, the numerical result obtained by Tremblay et al. [3] using the DNS is included in Fig. 8(b), from which we can see that the present numerical results is actually very close to the DNS prediction.

Fig. 7. Mean streamwise velocity component on the center line in the wake of the circular cylinder

Fig. 8. Mean streamwise and spanwise velocity components at five locations in the wake of the circular cylinder: (a)

streamwise; (b) spanwise 
The mean turbulent Reynolds stresses at different sections are also compared to the experimental data, as shown in Fig. 9. Based on the comparison, we can see that the present numerical results are accurate when the sections are not very close to the cylinder. However, at $x=1.06$, the LES model on the fine grid performs better than the DES model on the coarse grid, and the latter one under predicts the mean Reynolds stress components. Contours of instantaneous vorticity obtained by the LES model on the fine grid and the mixing-length based DES model on the coarse grid are shown in Fig. 10. The flow patterns for these two methods look similar. Two long shear layers separating from the cylinder and the development of the Karman vortex street are clearly seen in the figure. This also shows that the eddy content in the wake develops rapidly following boundary layer detachment, which indicates that the DES is also capable of simulating a flow experiencing massive separation.

Fig. 9. Mean Reynolds stress components at five locations in the wake of the circular cylinder: (a) streamwise Reynolds stress; (b) spanwise Reynolds stress; (c) Reynolds shear stress

Fig. 10. Instantaneous vorticity contour behind the circular cylinder in a cross-sectional plane: (a) Case LES_Mesh1; (b) Case MLD_Mesho

\subsection{Comparison of different RANS based DES models}

From the above study, it can be seen that by using the DES concept, the performance of the mixing-length model has been much improved. The developed mixing-length based DES model is also able to give superior results compared to the LES model on the same coarse grid. This brings the old mixing-length turbulence model back into focus, due to its easy implementation and high efficiency. However, a few new questions arise associated with the test of the DES concept. Can we implement the DES concept in other RANS turbulence models? Do they perform well in simulating the turbulence flows with massive separation? Which is the best among the different RANS based DES models? In order to answer those questions, different DES models are investigated by running the same flow past the circular cylinder at $\operatorname{Re}=3900$, and the numerical results for the global characteristics of the flow are summarized in Table 3. For the purpose of comparison, the experimental data and the numerical results by the LES model on the fine grid are also included in the table. The 0-equation mixing-length RANS model obviously gives the wrong results, and the 1-equation S-A RANS model and the 2-equation $k$ - $\omega$ RANS model over-predict the drag and the lift forces. Nevertheless, the results by different RANS based DES models are all acceptable, even though the maximum and minimum lift coefficients exhibit a little discrepancy because the lift force is sensitive to the stream turbulence. Furthermore, the LES results fall into the range between the 0 -equation and 1-equation based DES results.

Table 3. Hydrodynamic parameters of flow over the circular cylinder using different turbulence models at the grid Mesh0 
We also evaluate the performance of the various RANS models by comparing the mean Reynolds stress components with the experimental data, as shown in Fig. 11. At the station $x=1.06$ which is very close to the cylinder, all the RANS results for the spanwise Reynolds stress are over-estimated. More importantly, the experimental data indicates the peak appears twice in the Reynolds shear stress along the $y$ direction, but the RANS simulations fail to capture these two peaks. This suggests that the RANS models may not be very accurate in simulating flow in the near wake behind a circular cylinder.

Fig. 11. Comparison of mean Reynolds stress components at $x=1.06 \mathrm{~m}$ behind the circular cylinder using different RANS turbulence models at the grid Mesh0: (a) spanwise Reynolds stress; (b) Reynolds shear stress

The convergence of the three different RANS based DES models is also investigated at three different grids in terms of the mean drag coefficient, as shown in Table 4. It can be seen that the simulation at the grid "Mesh1" can provide the convergent results for all the three DES models, however, the convergent drag coefficient seems to be over-predicted compared to the experimental data. Among those results, the drag coefficient obtained by using the 0-equation based DES model is closest to the measurement. This discrepancy may be due to that the use of a Cartesian grid leads to a less accurate result in the boundary layer. In turbulence simulations, high resolution in the wall-normal direction is usually necessary to capture the detailed information in the boundary layer. However, the present Cartesian cut cell approach uses non-conforming grids to the body surface which is not easy to generate very fine meshes in the wall-normal direction. In addition, the wall function is not adopted in the near-wall region, which might be another reason for this discrepancy. The other possibility to cause such discrepancy is the simulation duration. In turbulence simulations, it is desirable to run the simulation as long as possible, so as to reach the final steady state. If the boundary layer is not fully developed, the resulting forces on the body may be influenced accordingly.

Table 4. Convergence of mean drag coefficient of flow over the circular cylinder with different computational meshes using various turbulence models

Furthermore, a dimensionless distance from the solid body $y^{+}$is a property that is always examined to ensure the resolution of the computational mesh adopted, which can be defined as

$y^{+}=\frac{\rho u_{*} y}{\mu}$

where $y$ is the typical size of the first near-wall grid and $u *$ is the shear velocity. If it is assumed that the flow is in local equilibrium, one can show [26]:

$u_{*}=C_{\mu}^{1 / 4} \sqrt{k}$

In the present numerical simulation, $y$ is taken as the size of the grid around the cylinder surface. As the 
turbulent kinetic energy $k$ is required in Eq. (20), the calculation is carried out for the 2-equation RANS based DES simulation to test this dimensionless distance. The results show that the $y^{+}$value varies between 16 and 17 with time at the coarse grid "Mesh0", and it is reduced to around 7 to 9 at the fine grid "Meshl". As in the DES simulation the RANS model is active near the solid surface, the $y^{+}$value for the fine grid "Meshl" at which the convergent results have been achieved lies in the acceptable range. However, the meshes are not distributed along the normal direction to the body surface due to the use of the Cartesian cut cell approach. The loss of accuracy is concentrated in the near wall region depending on the mesh size near the wall that cannot be adjusted easily to the shape of the obstacle.

Nevertheless, the laminar boundary layer in the flows investigated here may not have significant influence on the global fluid properties that are of the main interest in the present paper. In order to validate this statement, the mean spanwise velocity and streamwise Reynolds stress at two locations in the wake are investigated at three different grids. The corresponding results obtained by using the 0-equation RANS based DES model are chosen as an example for the purpose of demonstration, and shown in Fig. 12. Obviously, the results at these three grids are all agreeable with the experimental data. In fact, the "Mesho" result is very close to that at the grid "Mesh2", therefore, the following results for different RANS based DES models are given at the same grid "Mesh0".

Fig. 12. Comparison of mean spanwise velocity (a) and (c), and streamwise Reynolds stress (b) and (d) with different computational meshes using 0-equation RANS based DES turbulence model at: (a) and (b) $x=1.06 \mathrm{~m}$; (c) and (d) $x=3.0 \mathrm{~m}$ behind the circular cylinder

In order to assess different RANS based DES models, we compare the mean centerline velocity component in Fig. 13, and the mean velocity and Reynolds stress components at two different sections in Fig. 14 and Fig. 15 respectively. Fig. 13 indicates that the 1-equation DES model can provide the most satisfactory result compared to the experiment, while the 2-equation DES model leads to a smaller recirculation region. When the station is close to the cylinder at $x=1.06$ in Fig. 14, the 2-equation DES model seems to work well for the Reynolds stress components, however, it cannot capture the second peak in the spanwise velocity. At the station farther away from the cylinder in Fig. 15, we can see that the 2-equation DES model results are under-estimated for both the mean velocity and the Reynolds stress. Therefore, based on the overall performance, the 1-equation DES model is recommended, as it can give relatively accurate results for most cases at the coarse grid "Mesho". On the other hand, all the results obtained by using the 0 -equation DES model are also in the acceptable range. In addition, only $60 \%$ computer time is required in this model at the finest grid "Mesh2" compared to that in the 1-equation DES model. Therefore, if the computational efficiency is the main concern, the 0-equation DES model is also very promising due to the good balance between accuracy and efficiency.

Fig. 13. Comparison of mean centerline velocity component of the flow around the circular cylinder using different RANS 
based DES turbulence models

Fig. 14. Comparison of mean velocity and Reynolds stress components at $x=1.06 \mathrm{~m}$ behind the circular cylinder using different RANS based DES turbulence models: (a) spanwise velocity; (b) streamwise Reynolds stress; (c) spanwise Reynolds stress; (d) Reynolds shear stress

Fig. 15. Comparison of mean velocity and Reynolds stress components at $x=3.0 \mathrm{~m}$ behind the circular cylinder using different RANS based DES turbulence models: (a) spanwise velocity; (b) streamwise Reynolds stress; (c) spanwise Reynolds stress; (d) Reynolds shear stress

Lastly, Fig. 16 shows the contours of instantaneous vorticity obtained by two different RANS based DES models. From the comparison with Fig. 10, it can be seen that the 1-equation DES result is more consistent with the LES and 0-equation DES simulations, where we can notice massive separation in the wake region. However, the wake pattern predicted by the 2-equation DES model looks very regular, losing much detailed information about the flow separation, which again supports our recommendation made above.

Fig. 16. Comparison of instantaneous vorticity contour behind the circular cylinder in a cross-sectional plane using different RANS based DES turbulence models: (a) 1-equation DES; (b) 2-equation DES

The power spectrum distributions of the streamwise velocity components computed by the 1-equation RANS based DES turbulence model at three points on the centreline are shown in Fig. 17. It should be noted that the frequency $\omega$ is nondimensionalized by Strouhal shedding frequency $\omega_{0}$, and the power is nondimensionalized by the time interval square amplitude. It can be seen that the spectra peaks at these three locations on the centreline become very small, which is consistent with the observation in the experiment [32]. At the first point where the finer grids are adopted compared to the other region in the domain, the relatively good agreement between the numerical and theoretical results up to about $\omega / \omega_{0}=10$ indicates the correct representation of relatively small scales in the present numerical simulations. The agreement becomes worse at the other two points further downstream, and the $-5 / 3$ slope is reached over a relatively narrow band of frequencies, due to the coarse grids adopted in the region far from the body. Furthermore, the power spectrum at the finer mesh "Meshl" has also been compared with that in Fig. 17 (not present here), and the almost identical results are observed, which demonstrates that the present use of a low resolution in the near cylinder region seems not to affect the quality of the power spectrum. This remark again supports the idea that a very precise description of the boundary layer is not necessary to capture accurately the turbulent dynamics in the far wake.

Fig. 17. Frequency spectra of the streamwise velocity obtained by the 1-equation RANS based DES turbulence model at three downstream locations on the centerline: (a) $x / D=3.0$; (b) $x / D=5.0$; (c) $x / D=7.0$

A further interesting aspect that has been revealed by the current investigation employing various RANS based DES models is the border between the RANS and LES regions, as shown in Fig.18 where the RANS 
region is highlighted by the shaded area and the LES region elsewhere. As the RANS length scale is the wall normal distance, the RANS region is the same between the 0-equation and 1-equation RANS based DES models, and only the result of the 1-equation RANS based DES model is shown in Fig. 18(a). For these two DES models, the border is directly controlled by the grid fineness, which just surrounds the solid body surface. However, for the 2-equation RANS based DES model the RANS length scale is defined by the local turbulence model variables. Thus, the RANS length scale, and consequently the border location, is part of the solution and a function of space and time, as shown in Fig. 18(b). It should be pointed out that the border between the RANS and LES regions does not involve any discontinuities, as in DES simulations only one model is used in all regions and the border is simply defined where the limitation imposed in the DES concept is naturally triggered on or off.

Fig. 18. Instantaneous RANS region highlighted by the shaded area around the circular cylinder obtained by: (a) the 1-equation and (b) 2-equation RANS based DES turbulence models

\section{Conclusion}

A numerical model is developed in this paper to investigate the turbulent flow around three-dimensional bodies. Different turbulence models, including the LES model, RANS model and DES model are incorporated into a well-tested Navier-Stokes solver that adopts a finite volume method on a Cartesian cut cell grid. The Cartesian cut cell approach has already been proven to be a robust technique for geometrically complex computational domains with or without moving boundaries, which makes the developed numerical model capable of simulating complicated turbulent flows of engineering relevance. One important conclusion from the numerical study on the turbulent flow past square and circular cylinders is that the DES model, even constructed on the basis of the simplest mixing-length turbulence model, is able successfully to capture the critical behavior of the flow. On the same grid, the DES model can provide superior results compared to the RANS and LES models, based on the comparisons of the mean velocity and Reynolds stress components with the experimental data. The same flow past a circular cylinder is also used to examine the performance of the different RANS based DES models. The comparison leads to another important conclusion that the 1-equation S-A based DES model is very promising as it can give relatively accurate flow characteristics in the wake for this case. At the same time, if one must take the computational efficiency into account, the 0-euqation mixing-length based DES model is also recommended, in view of the good balance between accuracy and efficiency it can obtain.

\section{References}

[1] Evangelinos C, Lucor D, Karniadakis GE. DNS-derived force distribution on flexible cylinders subject to vortex-induced vibration. J Fluids Struct 2000;14:429-440.

[2] Ma X, Karamanos GS, Karniadakis GE. Dynamics and low-dimensionality of a turbulent wake. J Fluid Mech 
2000;410:29-65.

[3] Tremblay F, Manhart M, Friedrich R. DNS of flow around a circular cylinder at a subcritical Roynolds number with Cartesian grids. In: Proceedings of the $8^{\text {th }}$ European Turbulence Conference, Barcelona, Spain; 2000.

[4] Archer PJ, Thomas TG, Coleman GN. Direct numerical simulation of vortex ring evolution from the laminar to the early turbulent regime. J Fluid Mech 2008;598:201-226.

[5] Liang DF, Cheng L. Numerical modelling of flow and scour below a pipeline in currents Part I. Flow simulation. Coastal Eng 2005;52:25-42.

[6] Wanderley JBV, Souza GHB, Sphaier SH, Levi C. Vortex-induced vibration of an elastically mounted circular cylinder using an upwind TVD two-dimensional numerical scheme. Ocean Eng 2008;35:1533-1544.

[7] Spalart PR. Strategies for turbulence modelling and simulations. Int J Heat Fluid Flow 2000;21:252-263.

[8] Schmidt S, Thiele F. Comparison of numerical methods applied to the flow over wall-mounted cubes. Int J Heat Fluid Flow 2002;23:330-339.

[9] Kitagawa T, Ohta H. Numerical investigation on flow around circular cylinders in tandem arrangement at a subcritical Reynolds number. J Fluids Struct 2008;24:680-699.

[10] Liang C, Papadakis G. Large eddy simulation of pulsating flow over a circular cylinder at subcritical Reynolds number. Comput Fluids 2007;36:299-312.

[11] Spalart PR, Jou WH, Strelets M, Allmaras SR. Comments on the feasibility of LES for wings and on a hybrid, RANS/LES approach. In: Proceedings of $1^{\text {st }}$ AFOSR International Conference on DNS/LES; 1997.

[12] Spalart PR, Allmaras SR. A-one-equation turbulence model for aerodynamic flows. Rech Aerosp 1994;1:5-21.

[13] Travin A, Shur M, Strelets M, Spalart PR. Detached-eddy simulations past a circular cylinder. Int J Flow Turbu Combu 2000;63:293-313.

[14] Spalart PR. Strategies for turbulence modelling and simulations. Int J Heat Fluid Flow 2000;21:252-263.

[15] Bunge U, Mockett C, Thiele F. Guidelines for implementing detached-eddy simulation using different models. Aerosp Science Tech 2007;11:376-385.

[16] Hasama T, Kato S, Ooka R. Analysis of wind-induced inflow and outflow through a single opening using LES \& DES. J Wind Eng Industrial Aerody 2008;96:1678-1691.

[17] Squires KD, Krishnan V, Forsythe JR. Prediction of the flow over a circular cylinder at high Reynolds number using detached-eddy simulation. J Wind Eng Industrial Aerody 2008;96:1528-1536.

[18] Iaccarino G, Ooi A, Durbin PA, Behnia M. Reynolds averaged simulation of unsteady separated flow. Int J Heat Fluid Flow 2003;24:147-156.

[19] Breuer M, Jovicic N, Mazaev K. Comparison of DES, RANS and LES for the separated flow around a flat plate at high incidence. Int J Numer Meth Fluids 2003;41:357-388.

[20] Causon DM, Ingram DM, Mingham CG. A Cartesian cut cell method for shallow water flows with moving boundaries. Advances in Water Resources 2001;24:899-911.

[21] Liang QH, Zang J, Borthwick AGL, Taylor PH. Shallow flow simulation on dynamically adaptive cut cell quadtree grids. Int J Numer Meth Fluids 2007;53:1777-1799.

[22] Chung MH. Cartesian cut cell approach for simulating incompressible flows with rigid bodies of arbitrary shape. Comput Fluids 2006;35:607-623.

[23] Bai W, Mingham CG, Causon DM, Qian L. Finite volume simulation of viscous free surface waves using the Cartesian cut cell approach. Int J Numer Meth Fluids 2010;63:69-95.

[24] Nishino T, Roberts GT, Zhang X. Unsteady RANS and detached-eddy simulations of flow around a circular cylinder in ground effect. J Fluids Struct 2008;24:18-33.

[25] Wilcox DC. Reassessment of the scale-determining equation for advanced turbulence models, AIAA J 1988;26(11):1299.

[26] Ferziger JH, Perić M. Computational Methods for Fluid Dynamics. Springer: Berlin Heidelberg; 1999. 
[27] Norberg C. Flow around rectangular cylinders: Pressure forces and wake frequencies, J Wind Eng Industrial Aerody 1993;49:187-196.

[28] Durao DFG, Heitor MV, Pereira JCF. Measurements of turbulent and periodic flows around a square crosssection cylinder. Exp Fluids 1988;6:298-304.

[29] Bearman P, Obasaju E. An experimental study of pressure fluctuations on fixed and oscillating square section cylinders, J Fluid Mech 1982;119:297-321.

[30] Lourenco LM, Shih C. Characteristics of the plane turbulent near wake of a circular cylinder, a particle image velocimetry study. Private Communication; 1993 (data taken from Beaudan and Moin, 1994 [31]).

[31] Beaudan P, Moin P. Numerical experiments on the flow past a circular cylinder at a sub-critical Reynolds number. Report No. TF-62, Thermosciences Division, Department of Mech. Engineering, Stanford University; 1994.

[32] Ong L, Wallace J. The velocity field of the turbulent very near wake of a circular cylinder. Exp Fluids 1996;20:441-453.

[33] Norberg C. Effects of Reynolds number and low-intensity free stream turbulence on the flow around a circular cylinder. Publ. No. 87/2, Department of Applied Thermoscience and Fluid Mech., Chalmers University of Technology, Gothenburg, Sweden; 1987

[34] Nishino T, Roberts GT, Zhang X. Vortex shedding from a circular cylinder near a moving ground. Phys Fluids 2007;19:025103. 
Table 1. Mean drag coefficient and Strouhal number of flow over the square cylinder

\begin{tabular}{l|c|c|c|c|c}
\hline Case & $N x \times N y \times N z$ & Grid $\left(10^{6}\right)$ & $\Delta_{\min }\left(10^{-2}\right)$ & $C_{D}$ & $S t$ \\
\hline Exp. Norberg 1993 & - & - & - & 2.16 & 0.132 \\
Exp. Durao et al. 1988 & - & - & - & - & 0.133 \\
\hline LES_Mesh0 & $269 \times 160 \times 15$ & 0.646 & 1.44 & 0.677 & 0.117 \\
LES_Mesh1 & $309 \times 196 \times 15$ & 0.908 & 1.18 & 2.056 & 0.127 \\
LES_Mesh2 & $349 \times 230 \times 15$ & 1.204 & 1.00 & 2.147 & 0.127 \\
LES_Mesh1_NZ & $309 \times 196 \times 30$ & 1.817 & 1.18 & 2.177 & 0.137 \\
\hline MLM_Mesh0 & $269 \times 160 \times 15$ & 0.646 & 1.44 & 1.918 & 0.000 \\
MLM_Mesh1 & $309 \times 196 \times 15$ & 0.908 & 1.18 & 1.966 & 0.000 \\
\hline MLD_Mesh0 & $269 \times 160 \times 15$ & 0.646 & 1.44 & 2.210 & 0.137 \\
MLD_Mesh1 & $309 \times 196 \times 15$ & 0.908 & 1.18 & 2.450 & 0.127 \\
MLD_Mesh2 & $349 \times 230 \times 15$ & 1.204 & 1.00 & 2.481 & 0.127 \\
MLD_Mesh0_At & $269 \times 160 \times 15$ & 0.646 & 1.44 & 2.191 & 0.134 \\
\hline
\end{tabular}

Table 2. Mean drag coefficient and Strouhal number of flow over the circular cylinder

\begin{tabular}{l|c|c|c|c|c}
\hline Case & $N x \times N y \times N z$ & Grid $\left(10^{6}\right)$ & $\Delta_{\min }\left(10^{-2}\right)$ & $C_{D}$ & $S t$ \\
\hline Exp. Norberg 1987 & - & - & - & $0.98 \pm 0.05$ & $0.215 \pm 0.005$ \\
\hline LES_Mesh0 & $219 \times 172 \times 15$ & 0.565 & 2.00 & 0.762 & 0.264 \\
LES_Mesh0_NZ & $219 \times 172 \times 30$ & 1.130 & 2.00 & 0.835 & 0.249 \\
LES_Mesh1 & $279 \times 232 \times 15$ & 0.971 & 1.37 & 0.972 & 0.229 \\
LES_Mesh2 & $339 \times 292 \times 15$ & 1.485 & 1.04 & 1.056 & 0.220 \\
\hline MLM_Mesh0 & $219 \times 172 \times 15$ & 0.565 & 2.00 & 0.992 & 0.000 \\
MLM_Mesh1 & $279 \times 232 \times 15$ & 0.971 & 1.37 & 1.180 & 0.000 \\
\hline MLD_Mesh0 & $219 \times 172 \times 15$ & 0.565 & 2.00 & 0.984 & 0.229 \\
MLD_Mesh1 & $279 \times 232 \times 15$ & 0.971 & 1.37 & 1.138 & 0.210 \\
MLD_Mesh2 & $339 \times 292 \times 15$ & 1.485 & 1.04 & 1.133 & 0.205 \\
MLD_Mesh0_At & $219 \times 172 \times 15$ & 0.565 & 2.00 & 0.994 & 0.232 \\
\hline
\end{tabular}

Table 3. Hydrodynamic parameters of flow over the circular cylinder using different turbulence models at the grid Mesh0

\begin{tabular}{l|c|c|c|c}
\hline Case & $C_{D}$ & $C_{L_{\_} \max }$ & $C_{L_{\_} \min }$ & $S t$ \\
\hline Exp. Norberg 1987 & $0.98 \pm 0.05$ & - & - & $0.215 \pm 0.005$ \\
LES_Mesh1 & 0.972 & 0.332 & -0.334 & 0.229 \\
\hline 0-equation_RANS & 0.992 & 0.000 & 0.000 & 0.000 \\
0-equation_DES & 0.984 & 0.286 & -0.235 & 0.229 \\
\hline 1-equation_RANS & 1.160 & 0.841 & -0.843 & 0.234 \\
1-equation_DES & 1.046 & 0.439 & -0.573 & 0.225 \\
\hline 2-equation_RANS & 1.067 & 0.949 & -0.960 & 0.244 \\
2-equation_DES & 0.977 & 0.620 & -0.694 & 0.234 \\
\hline
\end{tabular}


Table 4. Convergence of mean drag coefficient of flow over the circular cylinder with different computational meshes using various turbulence models

\begin{tabular}{l|c|c|c}
\hline Case & Mesh0 & Mesh1 & Mesh2 \\
\hline 0-equation_DES & 0.984 & 1.138 & 1.133 \\
1-equation_DES & 1.046 & 1.278 & 1.278 \\
2-equation_DES & 0.977 & 1.272 & 1.232 \\
\hline
\end{tabular}

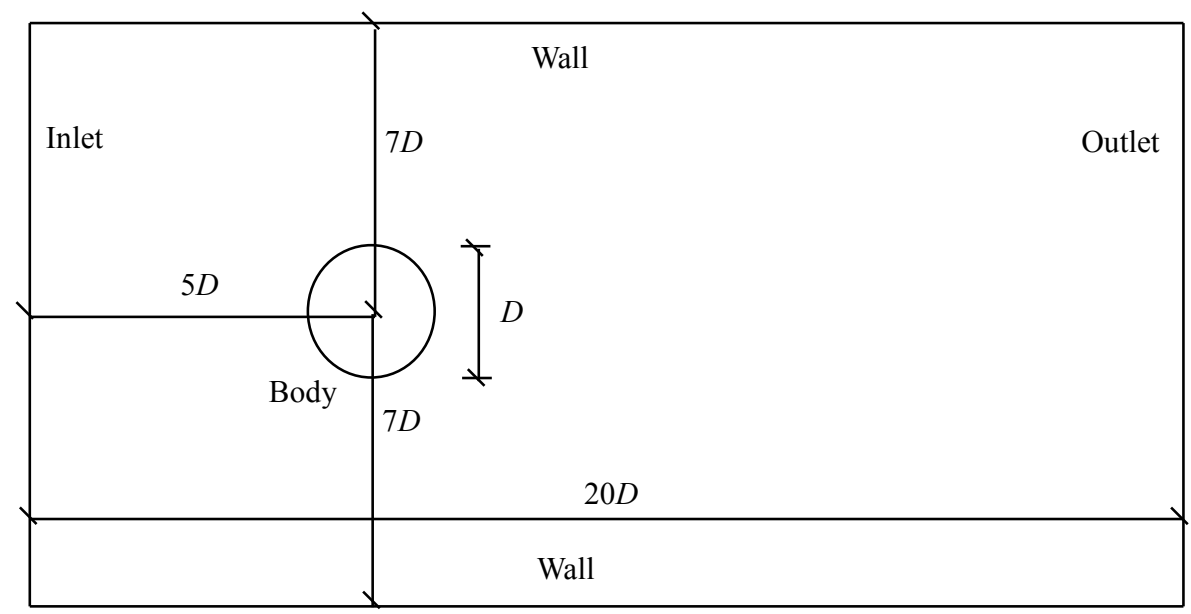

Fig. 1. Definition sketch of the flow domain

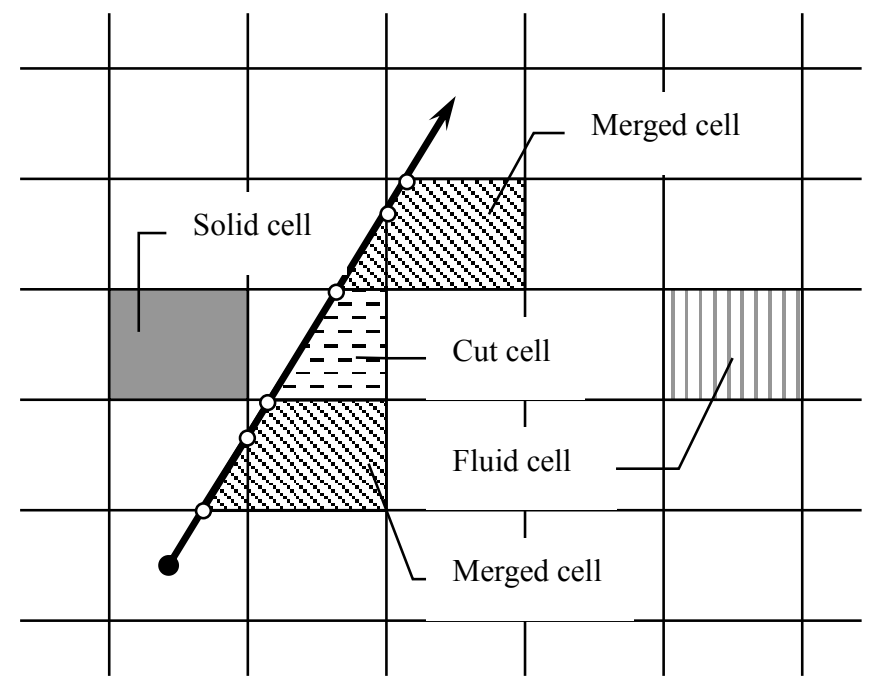

(a) 


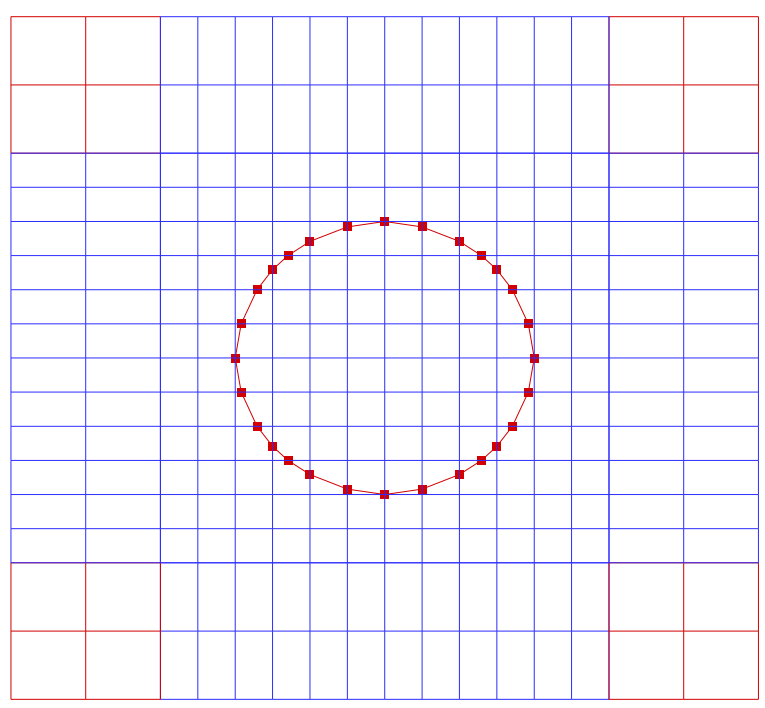

(b)

Fig. 2. (a) Sketch of definition for the cut cell mesh; (b) example cut cell mesh around a circular cylinder

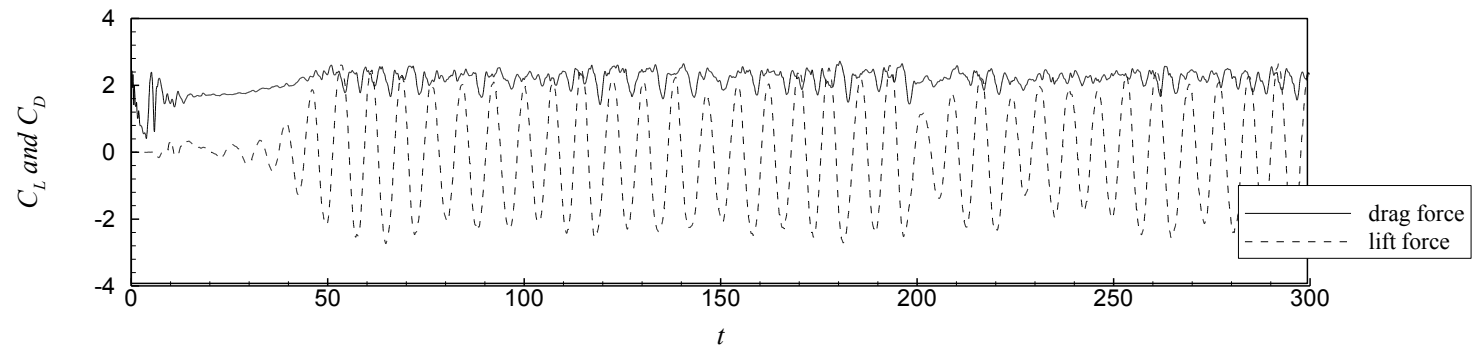

Fig. 3. Time history of drag and lift coefficients on the square cylinder for Case MLD_Mesho

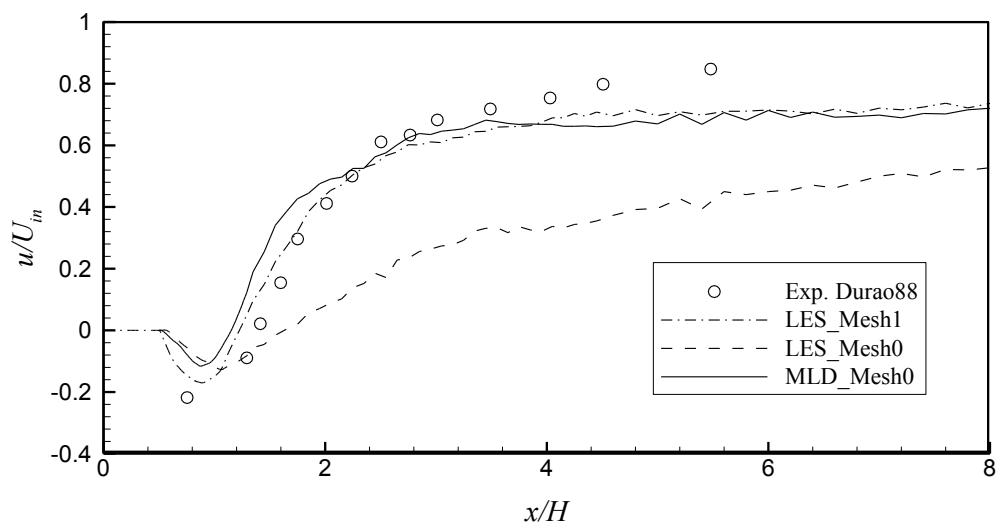


Fig. 4. Mean streamwise velocity component on the center line in the wake of the square cylinder

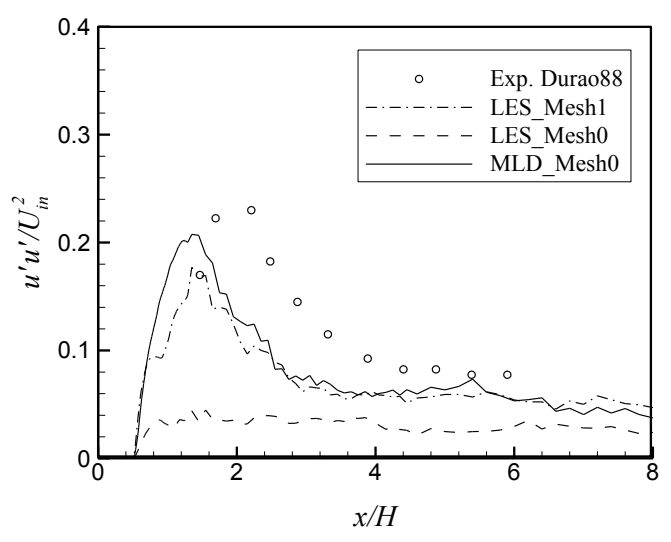

(a)

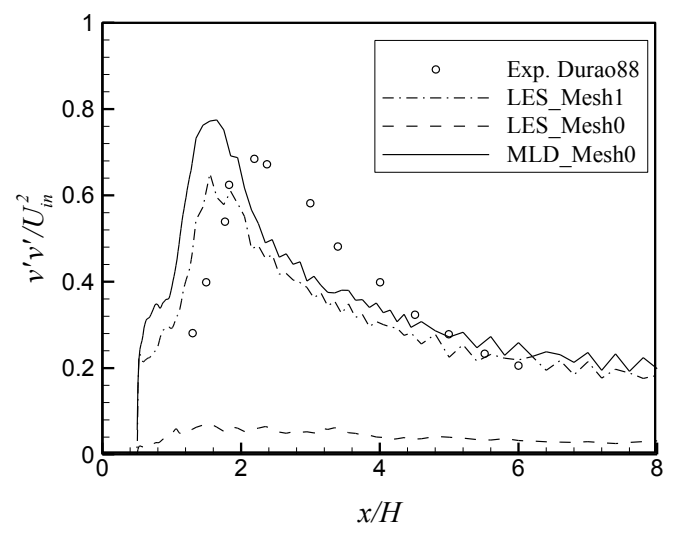

(b)

Fig. 5. Mean streamwise and spanwise Reynolds stress components on the center line in the wake of the square cylinder: (a) streamwise; (b) spanwise

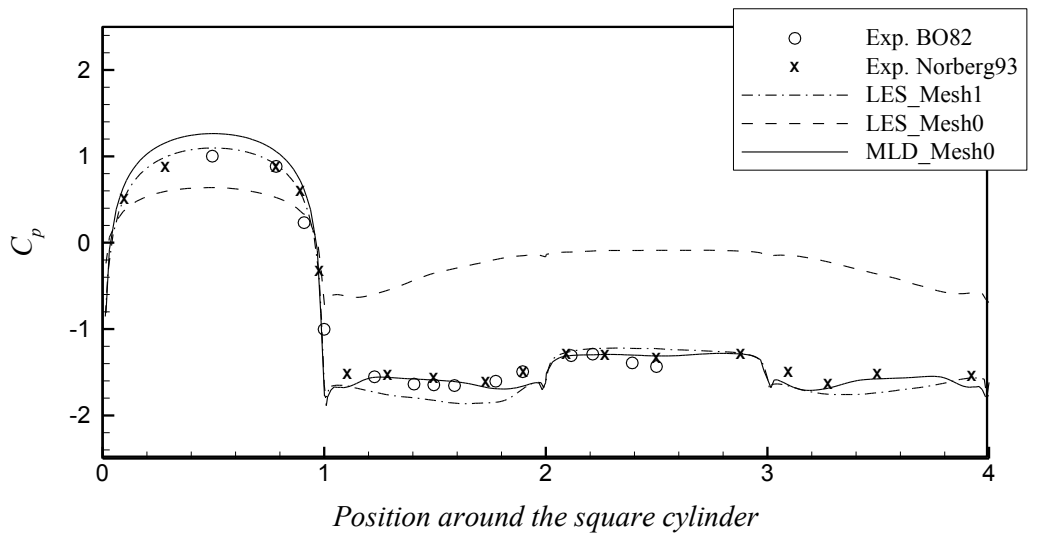

Fig. 6. Comparison of pressure coefficient around the square cylinder

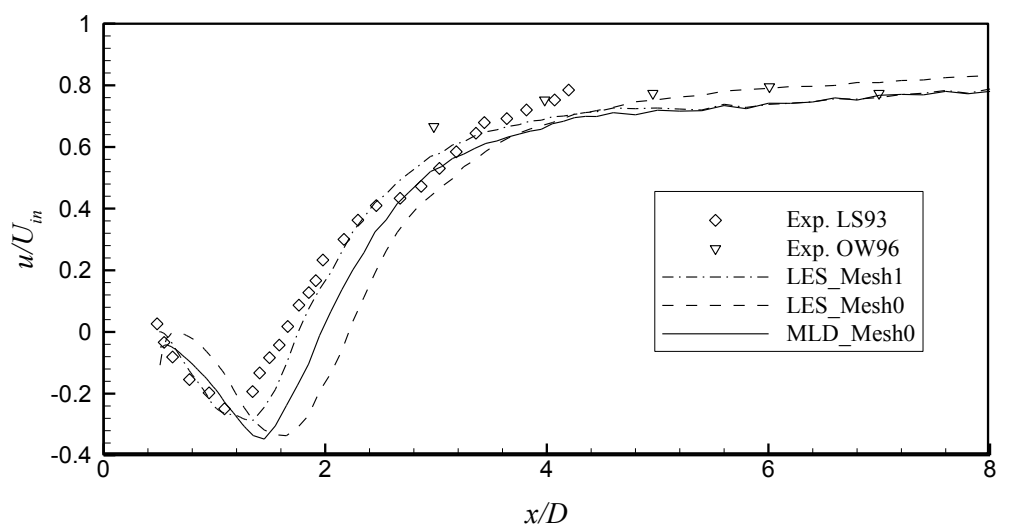

Fig. 7. Mean streamwise velocity component on the center line in the wake of the circular cylinder 


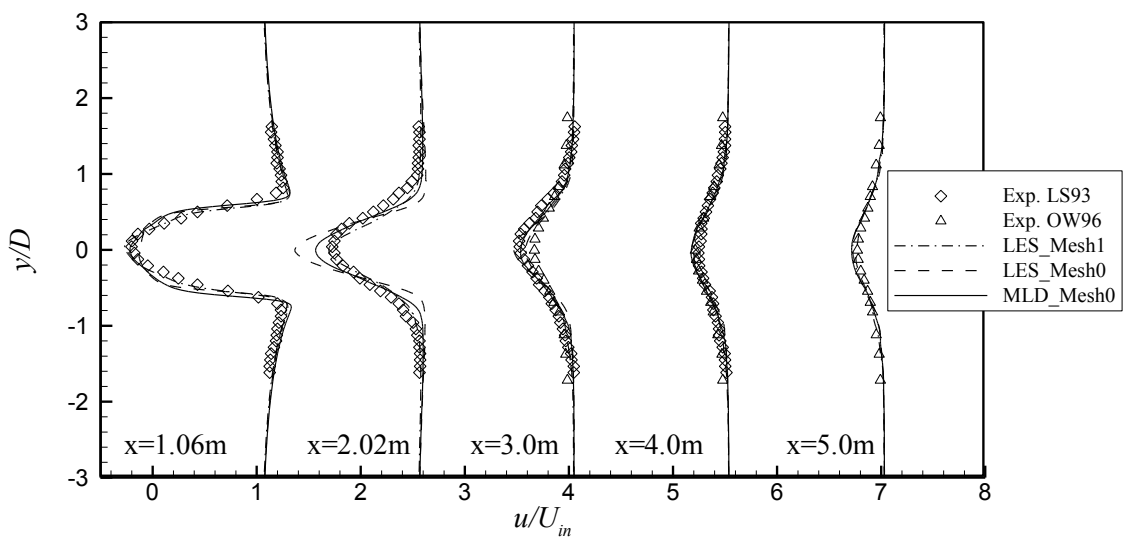

(a)

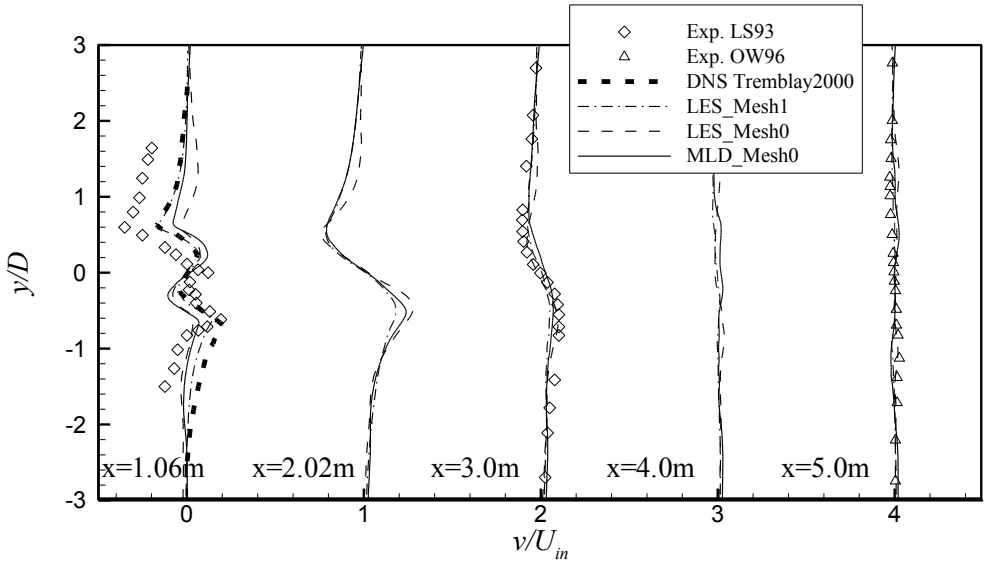

(b)

Fig. 8. Mean streamwise and spanwise velocity components at five locations in the wake of the circular cylinder: (a) streamwise; (b) spanwise

(a)

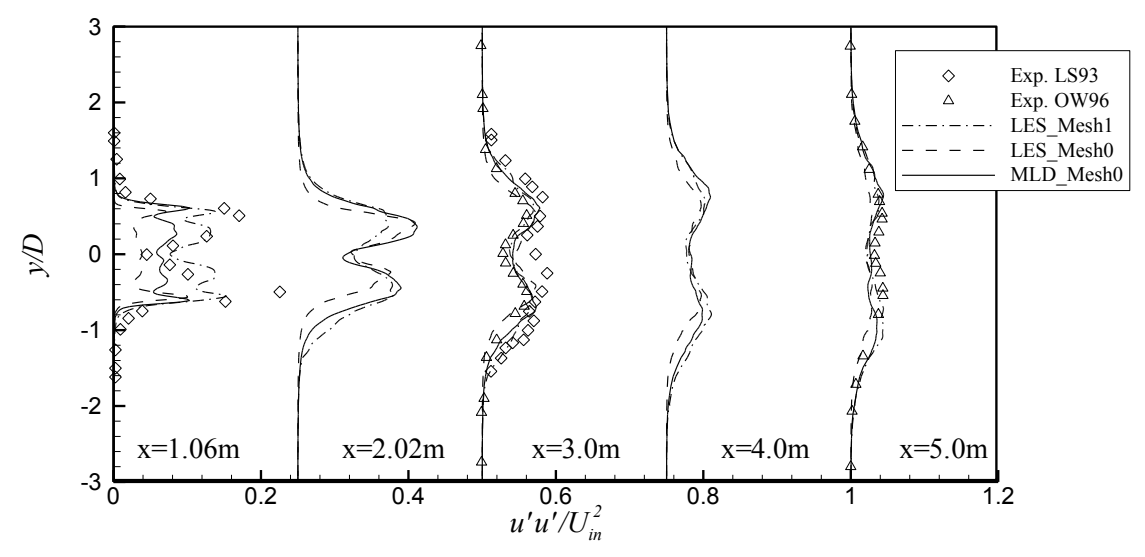


(b)

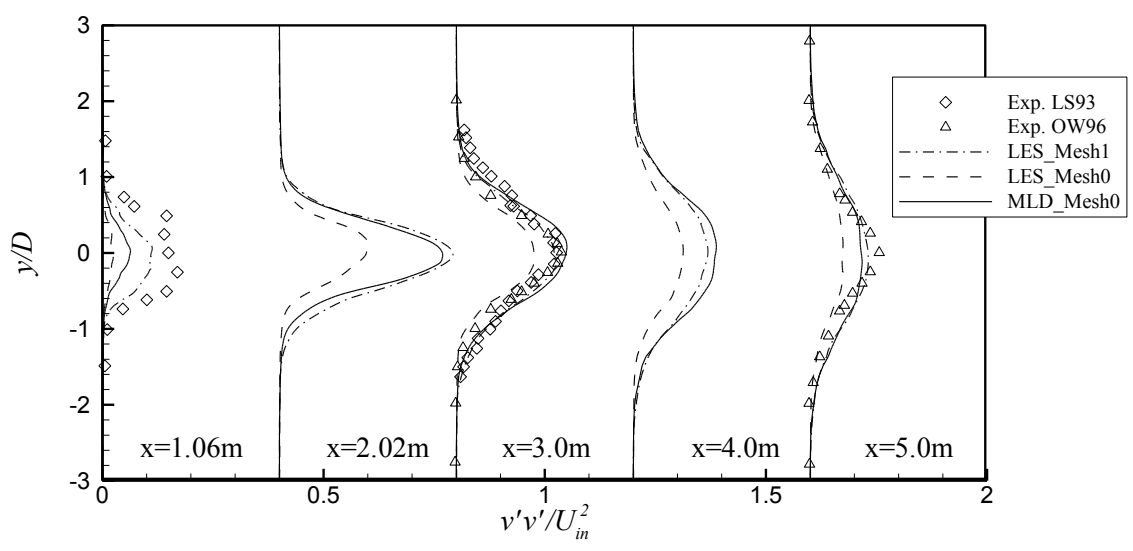

(c)

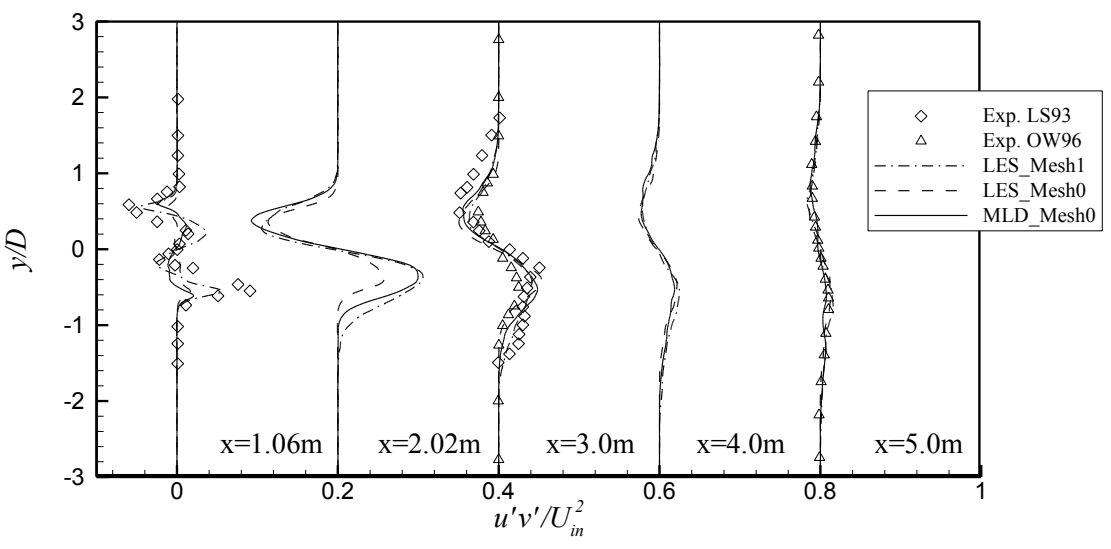

Fig. 9. Mean Reynolds stress components at five locations in the wake of the circular cylinder: (a) streamwise Reynolds stress; (b) spanwise Reynolds stress; (c) Reynolds shear stress

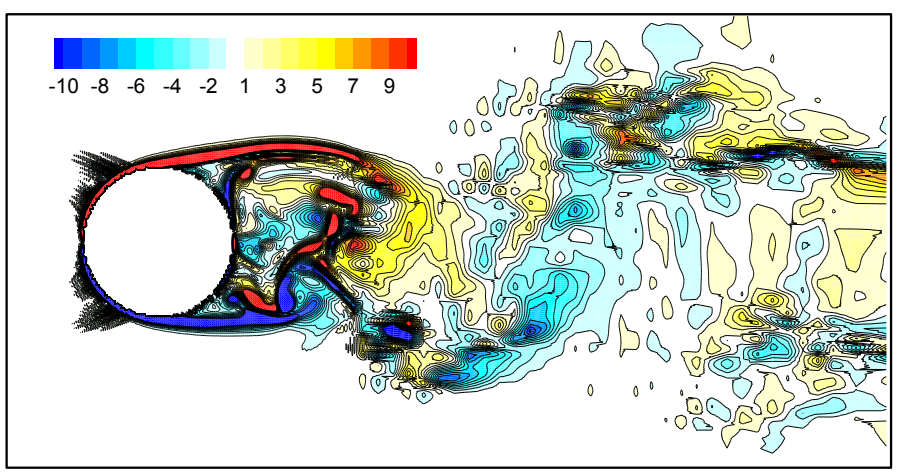

(a) 


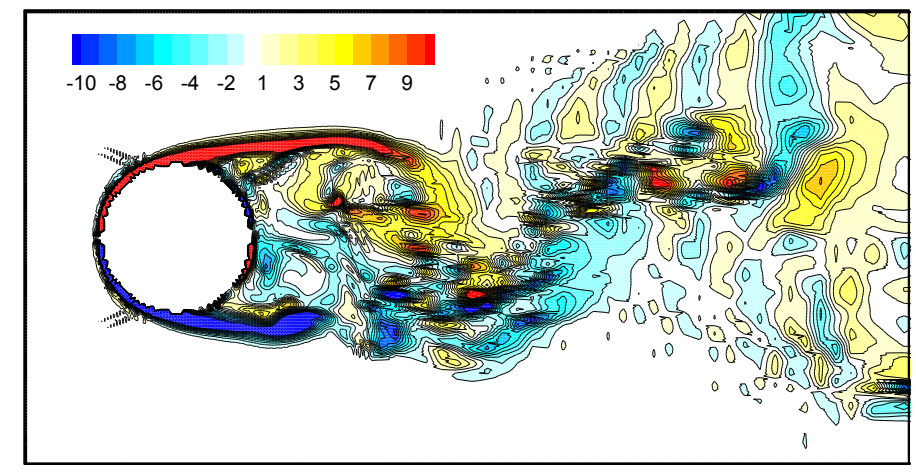

(b)

Fig. 10. Instantaneous vorticity contour behind the circular cylinder in a cross-sectional plane: (a) Case LES_Mesh1; (b) Case MLD_Mesho

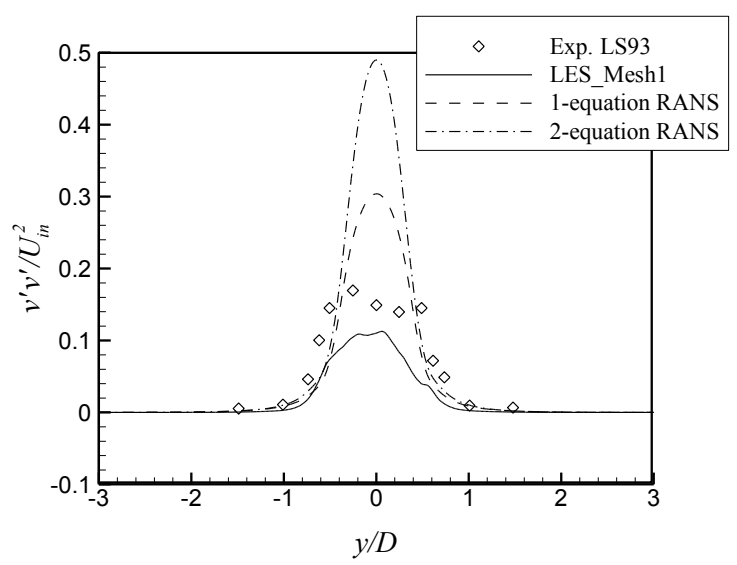

(a)

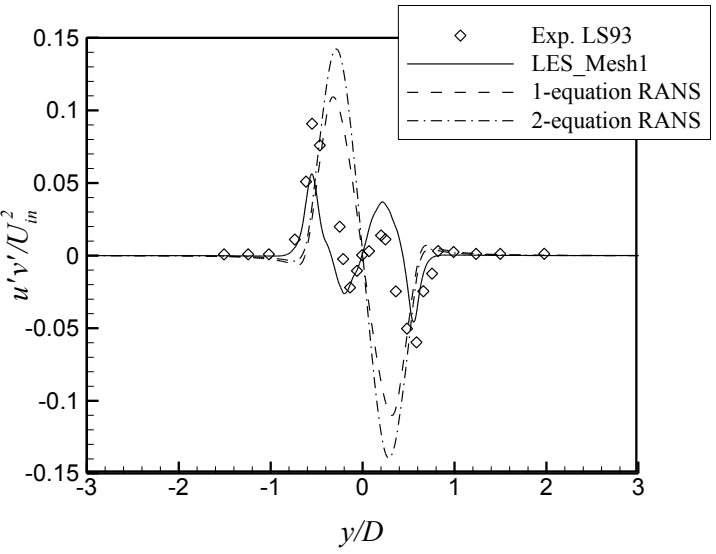

(b)

Fig. 11. Comparison of mean Reynolds stress components at $x=1.06 \mathrm{~m}$ behind the circular cylinder using different RANS turbulence models at the grid Mesh0: (a) spanwise Reynolds stress; (b) Reynolds shear stress

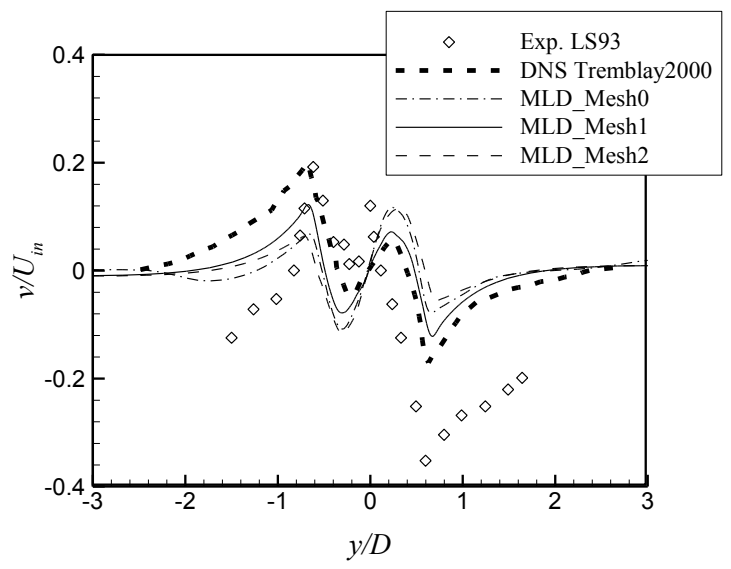

(a)

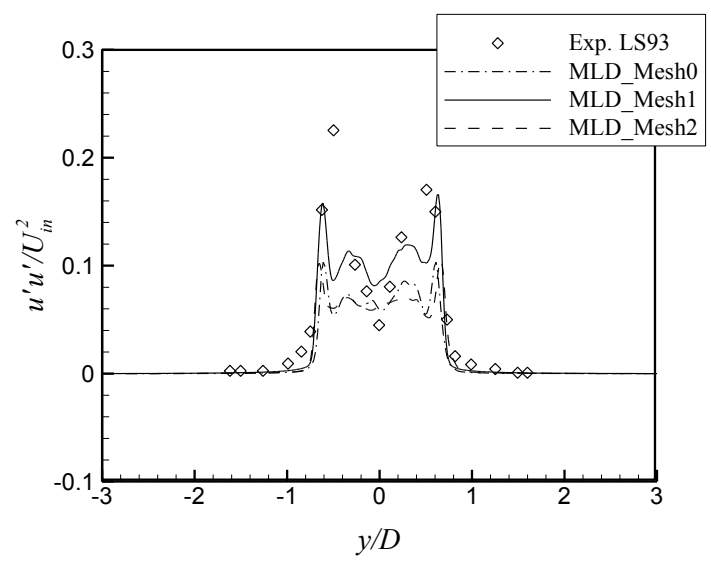

(b) 


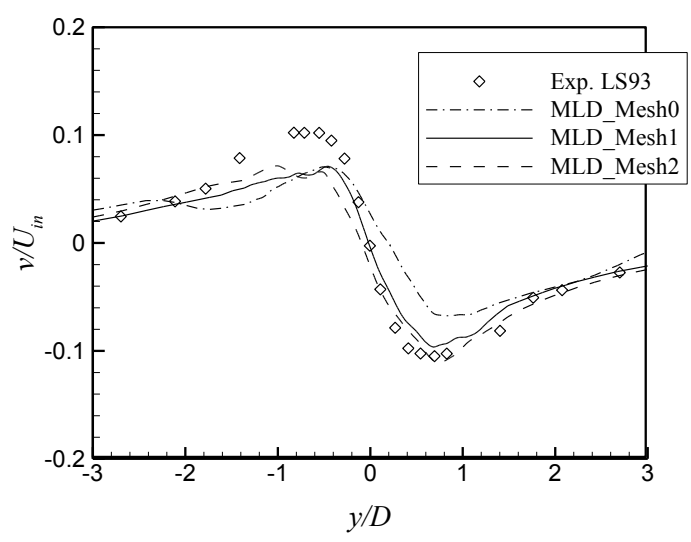

(c)

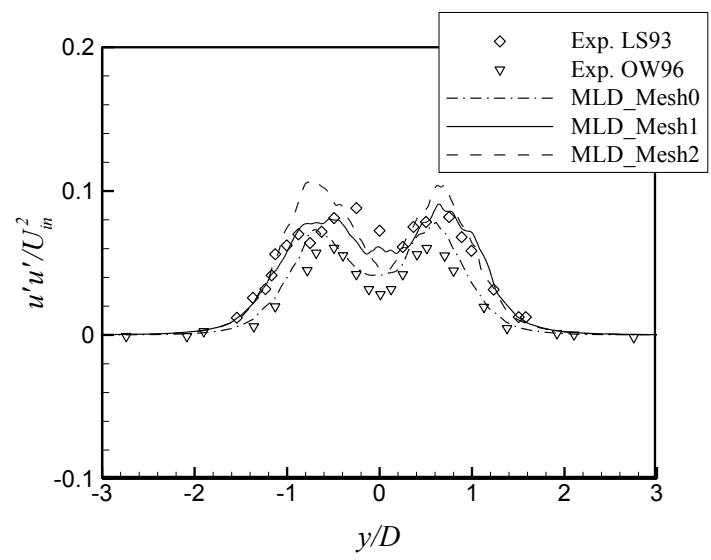

(d)

Fig. 12. Comparison of mean spanwise velocity (a) and (c), and streamwise Reynolds stress (b) and (d) with different computational meshes using 0-equation RANS based DES turbulence model at: (a) and (b) $x=1.06 \mathrm{~m}$; (c) and (d) $x=3.0 \mathrm{~m}$ behind the circular cylinder

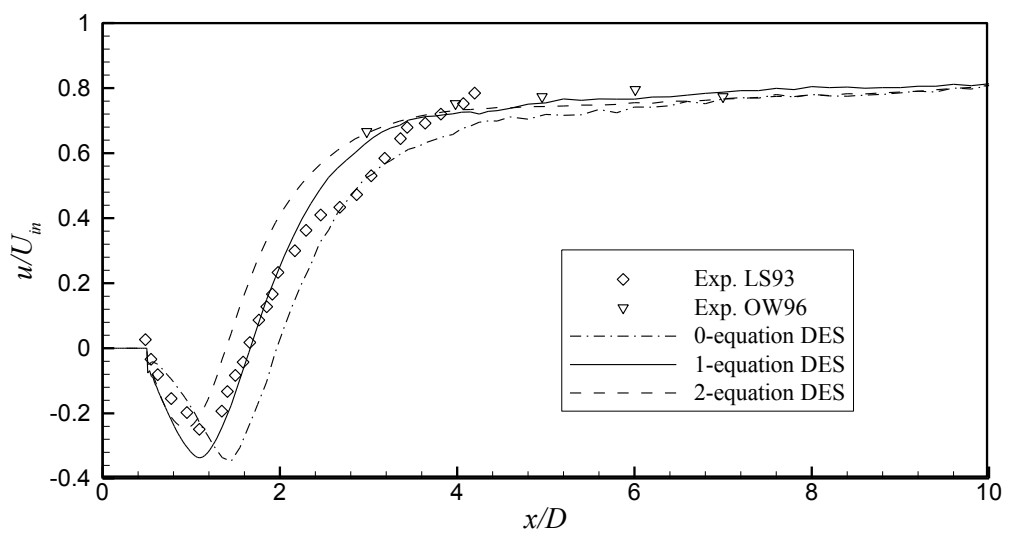

Fig. 13. Comparison of mean centerline velocity component of the flow around the circular cylinder using different RANS based DES turbulence models

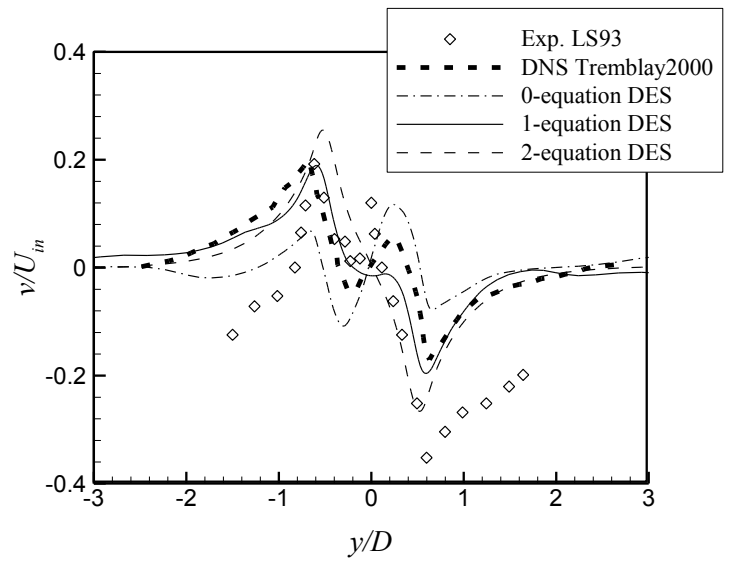

(a)

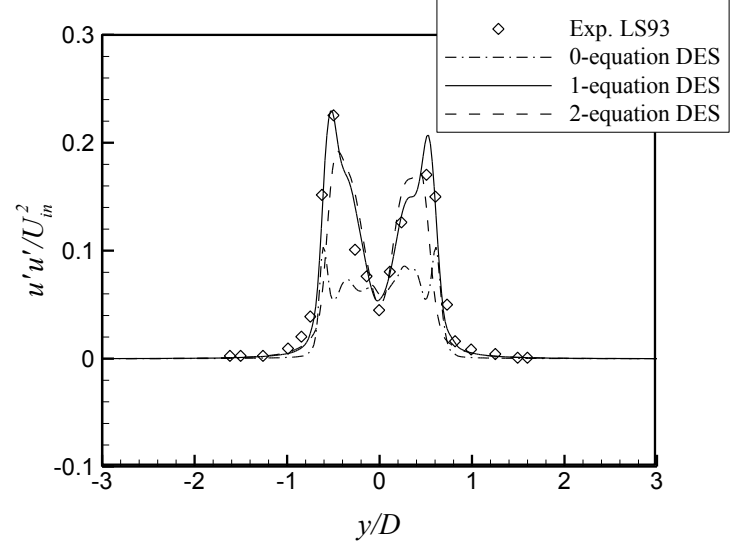

(b) 


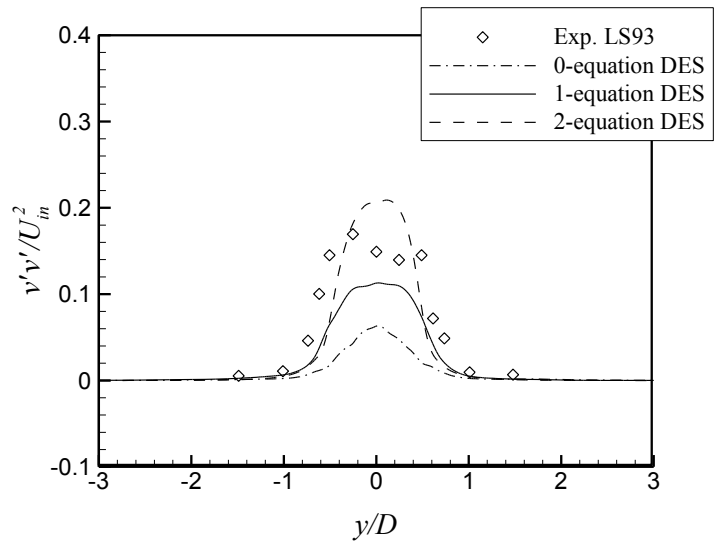

(c)

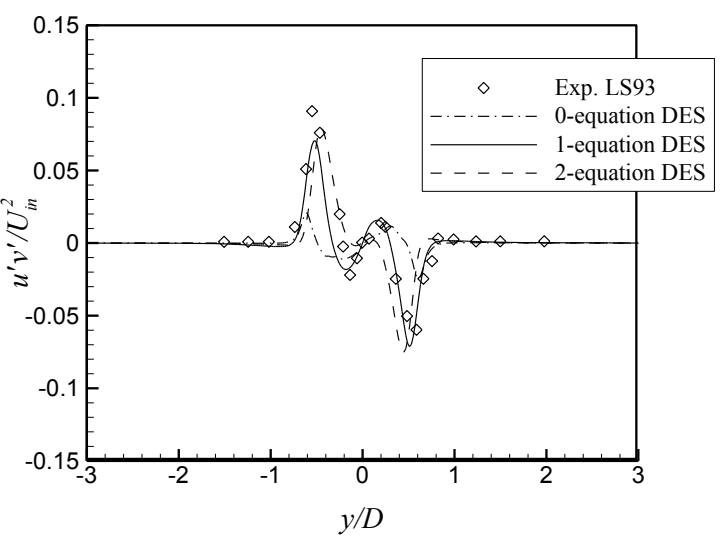

(d)

Fig. 14. Comparison of mean velocity and Reynolds stress components at $x=1.06 \mathrm{~m}$ behind the circular cylinder using different RANS based DES turbulence models: (a) spanwise velocity; (b) streamwise Reynolds stress; (c) spanwise Reynolds stress; (d) Reynolds shear stress

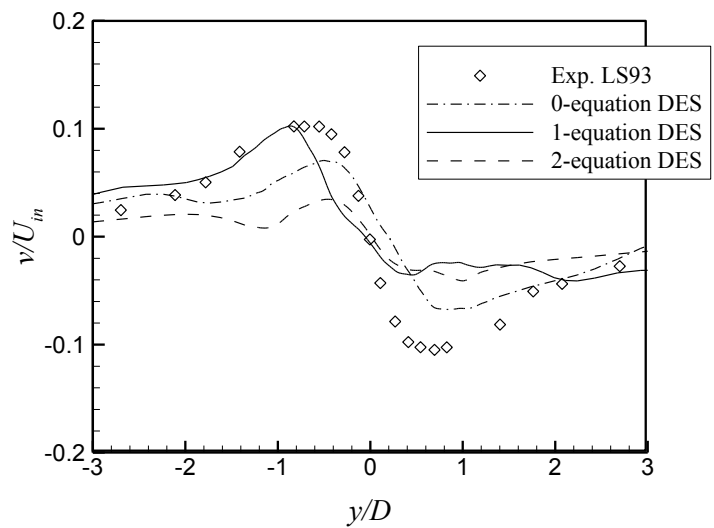

(a)

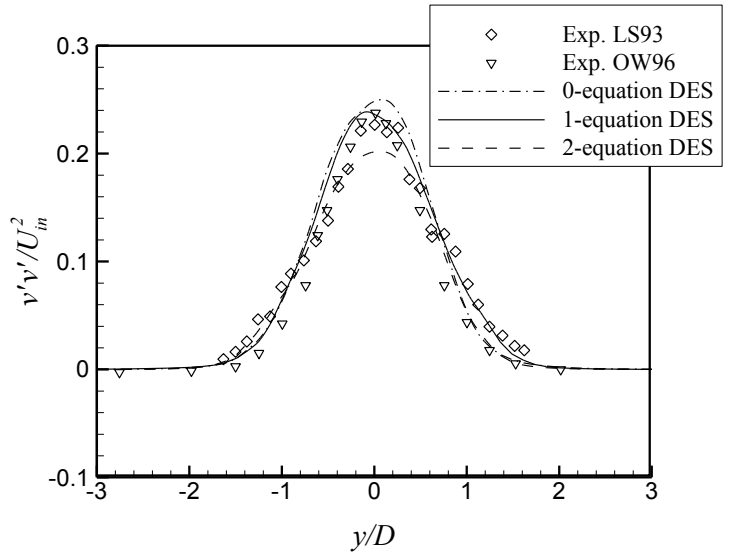

(c)

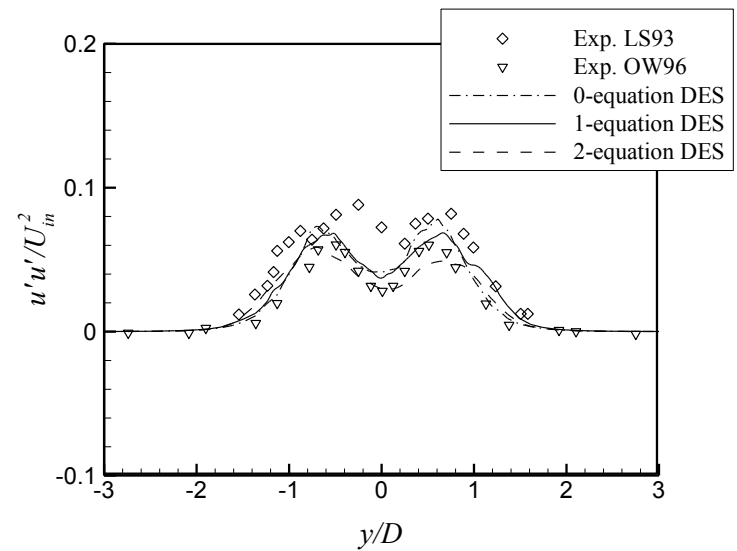

(b)

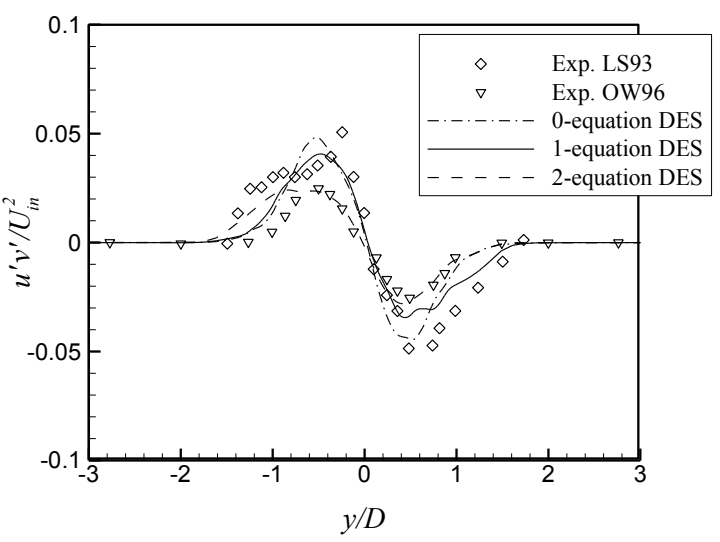

(d)

Fig. 15. Comparison of mean velocity and Reynolds stress components at $x=3.0 \mathrm{~m}$ behind the circular cylinder using 
different RANS based DES turbulence models: (a) spanwise velocity; (b) streamwise Reynolds stress; (c) spanwise Reynolds stress; (d) Reynolds shear stress

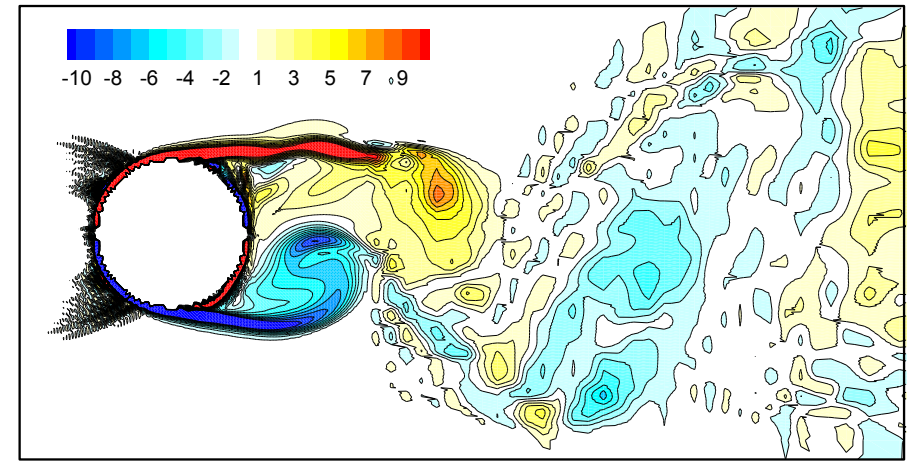

(a)

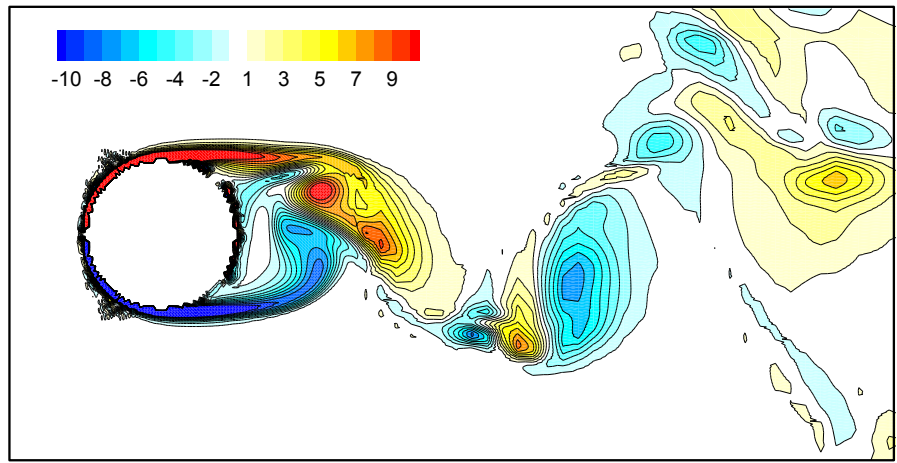

(b)

Fig. 16. Comparison of instantaneous vorticity contour behind the circular cylinder in a cross-sectional plane using different RANS based DES turbulence models: (a) 1-equation DES; (b) 2-equation DES 


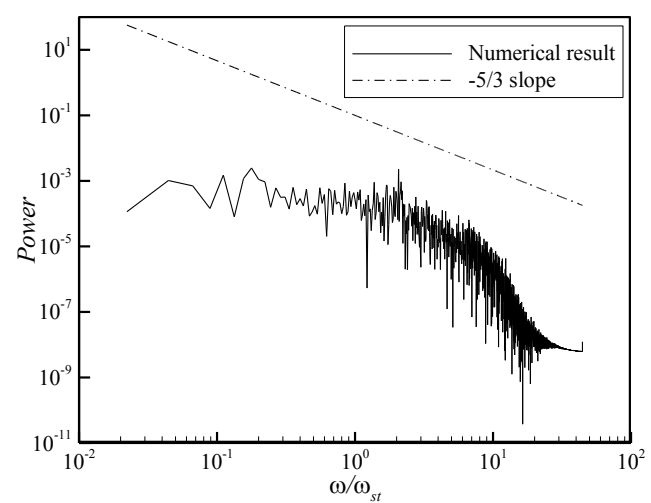

(a)

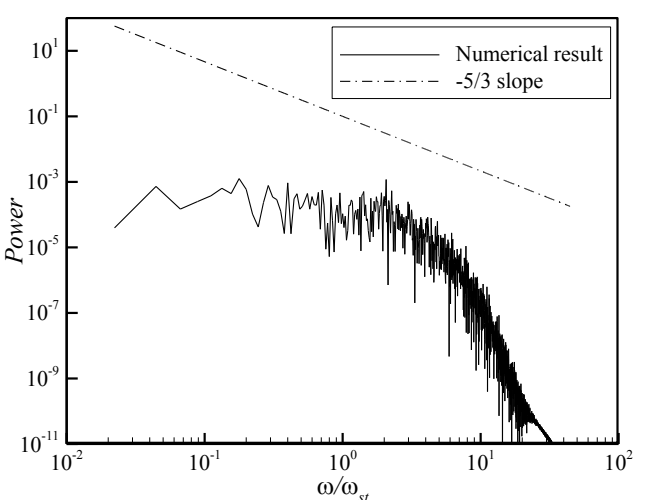

(b)

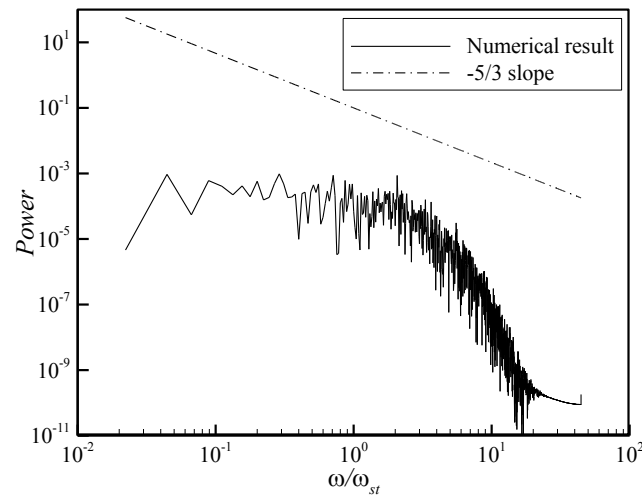

(c)

Fig. 17. Frequency spectra of the streamwise velocity obtained by the 1-equation RANS based DES turbulence model at three downstream locations on the centerline: (a) $x / D=3.0$; (b) $x / D=5.0$; (c) $x / D=7.0$

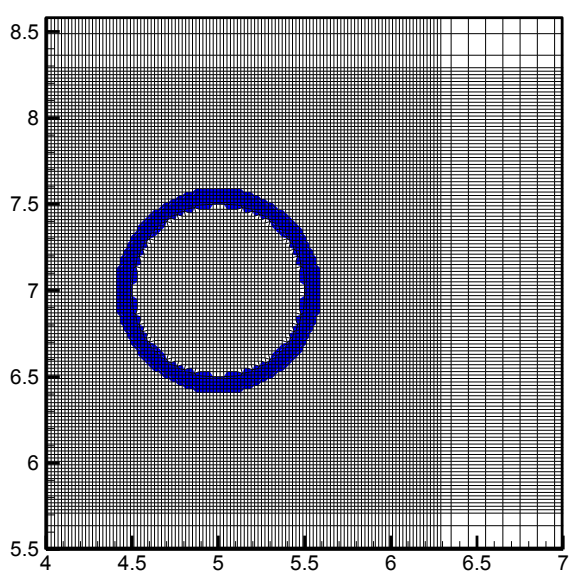

(a)

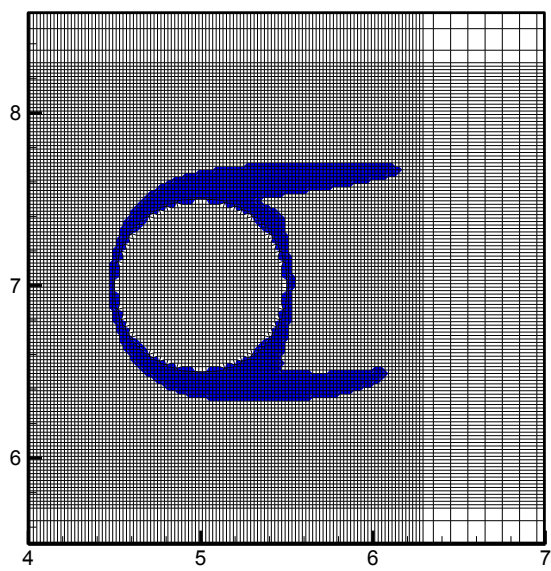

(b)

Fig. 18. Instantaneous RANS region highlighted by the shaded area around the circular cylinder obtained by: (a) the 1-equation and (b) 2-equation RANS based DES turbulence models 